\title{
AN ETHNOBOTANICAL SURVEY OF SPONTANEOUS PLANTS USED IN TRADITIONAL MEDICINE IN THE REGION OF AURES, ALGERIA
}

\author{
Karim BaZiz $^{1 *}$, Rim Tinhinene MaougaL ${ }^{2,3}$, AbdelKader Amroune $^{1}$ \\ ${ }^{1}$ Faculté de Médecine, Département de Pharmacie. Université Mostefa Ben Boulaïd, Batna 2, \\ Algérie. \\ *Correspondingauthor,E-mail:k.baziz@univ-batna2.dz \\ ${ }^{2}$ Institut de la Nutrition, de l'Alimentation et des Technologies Agro-alimentaires (INATAA), \\ département de Biotechnologie Alimentaire. Université Frères Mentouri, Constantine 1, Algérie. \\ ${ }^{3}$ Laboratoire de Génétique, Biochimie et Biotechnologie Végétale. Université Frères Mentouri, \\ Constantine 1, Algérie.
}

\begin{abstract}
.
Background: Like many regions in Algeria, the Aures abounds in an important ecological and floristic diversity. As a result, this region is the source of many plants used in traditional medicine. Despite this, the region is characterised by a lack of usable ethnobotanical data.

Methods: In current study, semi-structured questionnaires with 701 inhabitants from September 2018 to March 2019 were conducted. Data recorded were analysed using frequency of citation (RFC) to identify the well-known and most useful species in the area.

Results: This study allowed us to identify 112 medicinal spontaneous plants belonging to 47 families, of which Asteraceae, Lamiaceae, Amaranthaceae, Fabaceae, and Poaceae are the most represented and records new medicinal uses of 28 species. The leaves were the most used plant parts $(28.86 \%)$, and the decoction $(37.68 \%)$ represents the most frequently used mode of preparation. Also, the remedies were prepared essentially from single species $(81.15 \%)$ and were used mainly in the treatment of digestive disorders $(23.99 \%)$. The study has also shown that women, married people, the elderly, and illiterate people use traditional medicine much more than other categories.

Conclusion: This study provides an ethnobotanical survey of medicinal plants with their frequency of citation as well as the part used, the diseases treated and the modes of application in the Aures region. It indicates that the local population has fairly extensive empirical and traditional knowledge of herbal medicine. However, this knowledge is threatened mainly because of the mode of transmission that is based on oral tradition, and urgent transcription is needed to avoid the loss of this heritage.
\end{abstract}

Key words: ethnobotany; phytotherapy; spontaneous medicinal plants; traditional knowledge; vernacular plant name.

\section{INTRODUCTION}

Nowadays, if it is undeniable that modern medicine enjoys great success, particularly due to its excellent results, traditional medicine still retains a very important place in the African health system. Indeed, according to the World Health Organization (2013) up to $80 \%$ of African populations use medicinal plant preparations for treatment. This keen interest is explained by several factors including the efficiency of these treatments, the absence of undesirable side effects, their low cost, and socio-cultural habits of these populations (Tabuti et al. 2003; Muthu et al. 2006; Al-Adhroey et al. 2010). In the same way, the Algerian population remained attached to this knowledge which results from local cultural influences (Berber,
Maghreb, African) and the contribution of the Arab-Muslim academic medicine (Bouzabata \& Yavuz 2019).

The use of medicinal plants occupies an important place in traditional Algerian medicine and continues to be highly active, especially among local populations (Bouzid et al. 2017; Lazli et al. 2019). However, this knowledge has declined and is at risk of loss, essentially because recent economic developments combined with the modernization of infrastructure leaded young people to move away from rural localities, thereby breaking the oral transmission of this ancestral knowledge from older generations (Agarwal 1997). 
The geographical location of Algeria and its large surface area added to its soil and climatic diversity, have made it an area endowed with a great floristic diversity, concentrated globally in the northern regions (Vela \& Benhouhou 2007). This flora, valued at nearly 4,000 native taxa (Dobignard \& Chatelain 2010-2013) of which more than 600 plants are known to be used for medicinal and aromatic purposes (Mokkadem 1999), remains little explored in terms of traditional pharmacopoeia and ethnobotany.

The Aures region, a geographical entity that belongs to the Saharan Atlas domain and which undergoes combined climatic influences continental steppe, Mediterranean maritime, and mountain temperate, is rich in natural and botanical resources and consecrated by the presence of a large number of endemic, rare or threatened species (Yahi et al. 2008). Although several detailed studies relating to the diversity of local flora have been undertaken (Beghami \& De Bélair 2007; Beghami 2013; Beghami et al. 2013), it seems that very few studies concerning methods of traditional medicine preparations, parts used, and routes of applications of medicinal plants by local populations have been made.

In this context we have conducted an ethnobotanical study in the Aures region. This study consists of the design and analysis of a series of ethnobotanical surveys to identify the spontaneous plants used in traditional medicine by local populations and to inform about their importance in the therapeutic uses practised in the region.

\section{Materials AND Methods}

\subsection{Study site}

Our study was carried out in the Aures region

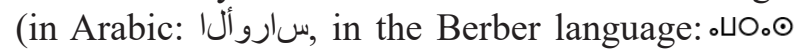
which is located in the northeast of Algeria,

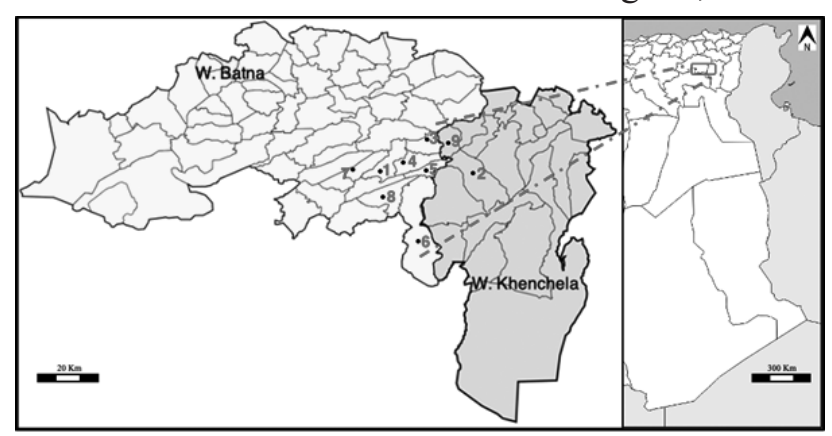

Figure 1. Map of the study area, located in eastern Algeria. The study sites are indicated by a number positioned on their location. 1: Arris, 2: Bouhmama, 3: Foum Toub, 4: Ichemoul, 5: Inoughissen, 6: Kimel, 7: Theniet El Abed, 8: T'kout, 9: Yabous some $470 \mathrm{~km}$ from the capital (Fig. 1). This region covers an area of $9,000 \mathrm{~km}^{2}$ between $34^{\circ} 30^{\prime}-35^{\circ} 5^{\prime}$ $\mathrm{N}$ and $05^{\circ} 45^{\prime}-07^{\circ} 20^{\prime} \mathrm{E}$. The relief is formed by a set of very contrasting mountain ranges including Djebel Chelia (2,327 m), Djebel El Mahmel (2,321 m), Djebel Lazreg (1,937 m), and Djebel Hmar khaddou $(1,842 \mathrm{~m})$ (Benmassouad et al. 2009). The region is formed of two geological units: the highest zones with limestone sandstone rocks of the Cretaceous age (Secondary) while the low altitude zones which present Tertiary and Quaternary terrains with sandstone facies, marly-limestone (Delartigue 1904).

A combination of various reliefs and diverse climatic conditions favoured the emergence of three bioclimatic stages of vegetation: a sub-humid stage, a semi-arid stage, and a stage at the upper arid limits at the southern piedmont of the massif (Beghami \& De Bélair 2007). As a result, the plant formations are very diverse and distributed according to the relief and the climate (Beghami et al. 2013): at high altitude, the ground cover is dominated by the Balansa broom (Cytisus balansae (Boiss.) Ball) and the thorny bupleurum (Bupleurum spinosum Gouan). At a lower level, the vegetation is dominated by the Atlas cedar (Cedrus atlantica (Endl.) Carrière). At the third level, the vegetation is affected by agropastoral practices and is dominated by the thuriferous juniper (Juniperus thurifera L.). At the lowest level, we observe the dominance of a forest formation composed of holm oak (Quercus ilex subsp. ballota (Desf.) Samp.), dimorphic ash (Fraxinus dimorpha Coss. \& Durieu), and thuriferous juniper (Juniperus thurifera L.).

\subsection{Population, sample, and data collection}

The reference population was all persons over 20 years of age born and living in the following localities: Arris, Bouhmama, FoumToub, Ichemoul, Inoughissen, Kimel, Theniet El Abed, T'kout, and Yabous (Fig. 1). Using a questionnaire, an open and semi-structured survey was carried out in a random sample of 701 people from September 2018 to March 2019.

The time spent on each interview was 20 minutes. The collected data concerned on the one hand the socio-demographic characteristics of the population like age, sex, profession, level of education, and family situation (Table 1) and on the other hand information concerning medicinal plants, their vernacular names, therapeutic uses, plant parts used, pharmaceutical forms used and the routes of admin- 
istration. The classification of diseases into pathological groups followed the International Classification of Primary Care 2 (ICPC 2015) used by the WHO as recommended by Staub et al. (2015).

The interviews were conducted in Chaoui or Arabic, as the case may require. Also, the botanical identification and family assignation were carried out at the Laboratoire de Biologie Végétale of the Department of Pharmacy at Batna-2 Mostefa Benboulaïd University by the first author, and voucher specimens deposited in its Herbarium. The identification was made by referring to the Algerian flora of Quezel and Santa (1962-1963) then updated according to the synonymic index of the North Africa flora (Dobignard \& Chatelain 2010-2013) and its online version, African Plant Database (http://www.villegen.ch/musinfo/bd/cjb/africa) and the World Checklist of Selected Plant Families (http://wcsp.science. kew.org/).

Data obtained were spread on an Excel spreadsheet and statistically analyzed (age, gender, education level, various proportions like plant families, plant parts used, methods of use, and frequency of citation, FC). This frequency, used to assess the importance of species (Trotter \& Loghan 1986) was computed using the following formula:

$F C=\Sigma U i / N \times 100$ where $U i$ is the number of informants who cited the species and $N$, the total number of informants.

\section{RESULTS AND DISCUSSION 2.1. Informants profile}

The use of traditional medicine and phytotherapy is frequent among the population of Aures and often remains the first treatment to which they resort. Indeed, of the 701 questioned persons, 595 people representing $84.88 \%$ used plants in medication, and thus 106 persons were excluded because they did not use plants to treat themselves. The above result coincides with this mentioned in the World Health Organisation (2013) report on strengthening the role of traditional medicine in African health systems, where more than $80 \%$ of the African population is reported to use traditional medicine for healing purposes.

Among these users, 399 individuals (67.06\%) use herbal medicine alone. For these persons, the recourse to traditional medicine is a question of availability and preference for plants to drug treatments $(60.67 \%)$, unavailability of conventional drugs (19.16\%), cost of conventional drugs (16.80\%), and finally disillusions linked to conventional drugs side effects $(3.36 \%)$. In other words, the practice of phytotherapy in the Aures has a more traditional and cultural dimension than an economic one. This corroborates the findings of Sanogo (2006), who considered that it is, first of all, the socio-cultural habits of the populations that explain the use of traditional practices based on medicinal plants followed by the lack of conventional drugs, insufficient health care, and finally the high cost of drugs.

Moreover, 196 individuals (32.94\%) use both phytotherapy and modern medicine. According to Olivier de Sardan (1995), in a favourable socio-economic context, patients combine traditional and modern medicine remedies to increase and maximize the chances of recovery.

The survey also provides very interesting information on the socio-demographic characteristics of users of medicinal plants in the region (Table 1). Thus, concerning gender and marital status, an intra-category comparison gives a percentage of $70.08 \%$ women and $70.25 \%$ married people among the users of herbal medicines. These results find their explanation similar to the Chaouia rural family (Boutamine 2018) where the role of household management, of "caring for oneself and others" and "controlling the family budget" is mainly assigned to women to reduce the material costs induced by the doctor and the drugs. These results are in agreement with those obtained at a national scale by Bouallala et al. (2014), Souilah et al. (2018) and Chohra and Ferchichi (2019), and north African scale by Mehdioui and Kahouadji (2007), Jdaidi and Hasnaoui (2016) and Chaachouay et al. (2019) who also stressed the important role of married women in traditional medicine.

In terms of age, the use of phytotherapy and traditional medicine is the prerogative of the oldest (Table 1). In this respect, the 60 years old is the over-represented group with $31.26 \%$ of users. The age groups [50-59], [40- 49], [30-39] and [20-29] then come with a percentage of $25.54 \%, 19.66 \%$, $15.79 \%$ and $7.73 \%$ respectively. We also noted that $55.96 \%$ of plant users refer to their own experiences and those of ancestors in the use of medicinal plants as remedies, $24.70 \%$ solicit herbalists while $18.65 \%$ consults books and radio/television programs specializing in herbal medicine. Finally, a marginal proportion of $0.69 \%$ consults a pharmacist. These results indicate that the practice of phytotherapy as well as the acquisition of knowledge on the therapeutic virtues of plants is done traditionally and empirically. This profile, similar to that observed by Hamel et al. 
(2018) and Souilah et al. (2018) confirms that accumulated experience and family knowledge are the main sources of information. Furthermore, the small percentage of the youngest people, probably due to their reluctance or lack of interest in traditional medicine, shows a break in the transmission of knowledge as described by Anyinam (1995). Another possible explanation for this is that young people often have other activities and have very little time to spend with the older knowledgeable persons. The information acquired by the youngest deteriorates as they engage in other activities.

Table 1. Demographic profile of users of medicinal plants interviewed $(\mathrm{N}=595)$

\begin{tabular}{l|r}
\hline Variables & Proportion (\%) \\
\hline Age & 7.73 \\
$20-29$ years & 15.79 \\
$30-39$ & 19.66 \\
$40-49$ & 25.54 \\
$50-59$ & 31.26 \\
\hline 60 & \\
\hline Gender & 70.08 \\
Female & 29.92 \\
Male & 70.25 \\
\hline Marital status & 29.75 \\
Married & \\
Single & 41.34 \\
Educationallevel & 25.04 \\
Illiterate & 22.85 \\
Primary & 10.77 \\
Secondary & \\
University & \\
\hline
\end{tabular}

Interestingly, the use of medicinal plants decreased with the level of education of plant users (Table 1). Indeed, the survey shows that the majority of informants are illiterate $(41.34 \%)$, the rest being made up of individuals of primary level $(25.04 \%)$, secondary level $(22.85 \%)$, and university level $(10.77 \%)$. This suggests that subjects without education or poorly educated are deprived of material resources and, in this case, they cannot access conventional drugs. This might also be attributed to the fact that the academics would show little confidence in traditional medicine and are more attracted by modern medicine. This outcome is contrary to that of a similar study conducted by Chohra and Ferchichi (2019) in the neighboring region of Belezma who found that most users are not illiterate. Further, the high illiteracy rate among medicinal plant users constitutes a danger of degradation of this flora and may seriously compromise the sustainability of the natural resources due to the over-harvesting. Analysing this problem, Rebbas et al. (2012) concluded that 'an integrated strategy for conservation of biodiversity must be installed'. This might be done by increasing ethnobotanical studies which allow better management of gathering of plants or by promoting the cultivation of certain species with great medicinal and economical potential.

\subsection{Plant species and botanical families}

As indicated in Table 2, a total of 112 spontaneous medicinal plant species were collected and identified by the researchers from the study area. Those species were divided into 106 genera and 47 families. Seven species belong to the Gymnosperms (four species from the Cupressaceae family, two species from the Pinaceae, and one species from the Ephedraceae). Angiosperms are represented by 8 monocotyledons $(7.20 \%)$ and 96 dicotyledons $(86.48 \%)$. Among the 47 families, five families alone hold 47 species which account for $41.96 \%$ of all inventoried medicinal plants. Among them, Asteraceae was the most dominant family ( 22 genera; 25 species) followed by Lamiaceae $(6 ; 7)$, Amaranthaceae $(5 ; 5)$, Fabaceae $(5 ; 5)$, and Poaceae $(5 ; 5)$ respectively. On the other hand, 25 families are represented by a single species (Fig. 2). The high proportion of these botanical families compared to the others could be attributed to their specific richness at the local level due to the ecological factors that favour the development and adaptation of these families. Indeed, the Asteraceae, the Poaceae, the Fabaceae, and the Lamiaceae alone represent more than $42 \%$ of all the species listed locally (Beghami 2013). This also provides a good indication of deep knowledge of plants and the development of this flora by the local populations for their different needs. Similar studies were conducted locally (Chohra \& Ferchichi, 2019) or national scale (Ould el hadj et al. 2003; Hadjaiji-Benseghier \& Derridj 2013; Lazli et al. 2019) also revealed the strong contribution of these families to the traditional Algerian pharmacopoeia. These studies show that even if the main families are the same overall, the most represented family is different from one study to another. This disparity in the results is attributed to the geographic diversity of the study areas and the difference between the populations studied (Kadri et al. 2018). Furthermore, according to many authors, such as Hernández et al. (2012), Asteraceae, Poaceae and Fabaceae are considered among the most exploited families in traditional medicine. Dhaou et al. (2010) explain this popularity by their wide range of bioactive compounds. 


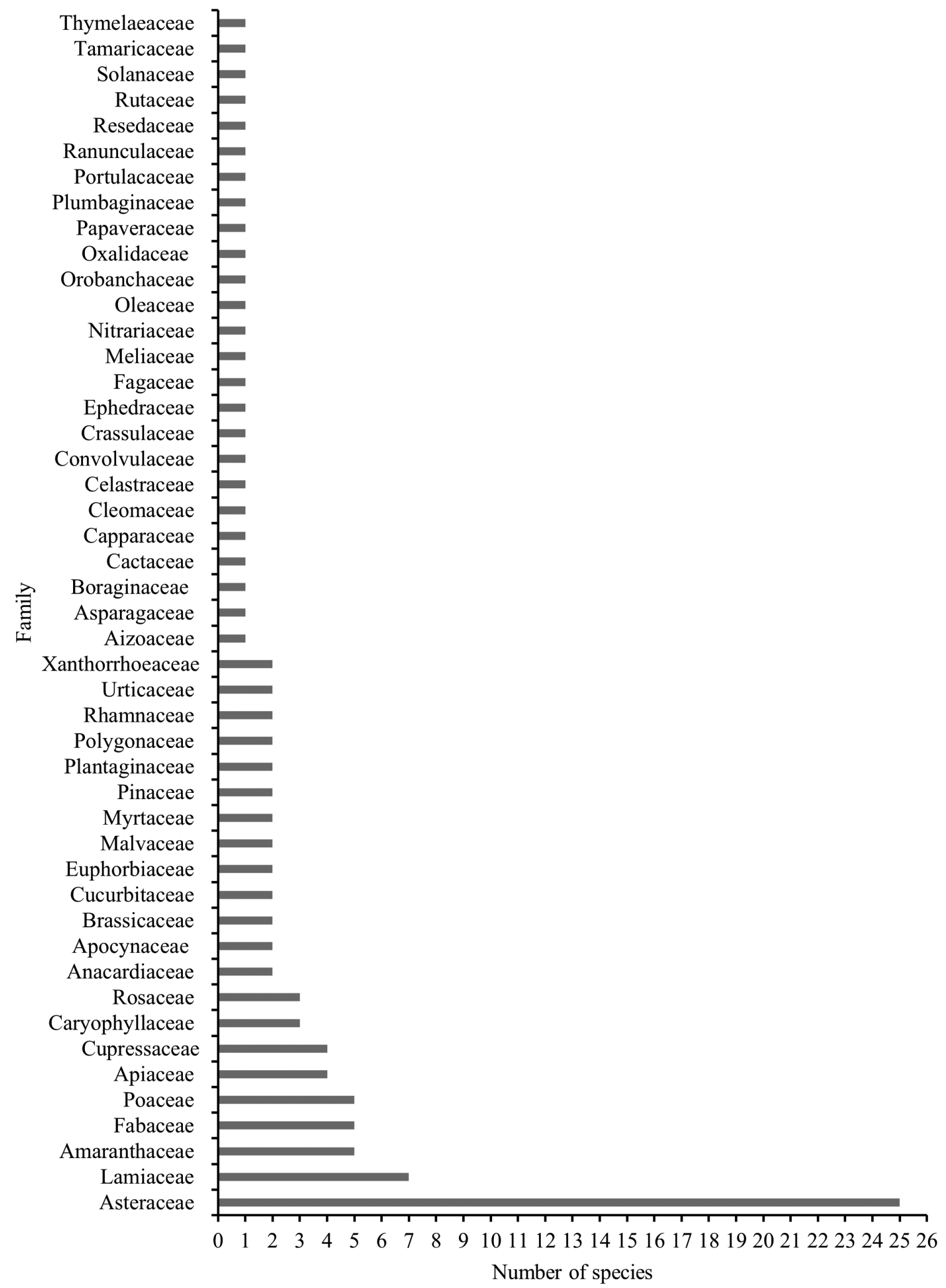

Figure 2. Distribution of species by botanical family. 
Of the plant listed in this survey, the most cited species (frequency of use $>10 \%$ ) were Rosmarinus officinalis with a frequency of $24.20 \%$ corresponding to 144 citations, followed by Pistacia lentiscus (23.69\%; cited 141 times), Marrubium vulgare (21.69\%; 129), Pinus halepensis (20.84\%; 124), Artemisia Campestris (20.50\%; 122), Cupressus sempervirens (20.02\%; 119), Artemisia herba-alba (19.83\%; 118), Calendula officinalis $(14.80 \% ; 88)$ and finally, Juniperus phoenicea (13.94\%; 83). The importance of these species in the traditional Algerian pharmacopoeia is well known (Baba Aissa 2000; Beloued 2005) and various phytochemical investigations on these plants have been undertaken and confirmed their extraordinary medicinal properties (Djeridane et al. 2006; Atmani et al. 2009; Bezza et al. 2010; Rached et al. 2010; Bouterfas et al. 2014).

Our results describe new records of traditional use for 28 species (highlighted in bold; Table 2). In particular two species should be underlined: Xanthium orientale subsp. italicum $(=X$. italicum) whose use for medicinal purposes is not known in Algeria and Ampelodesmos mauritanicus which is identified as a medicinal plant for the first time.

The new records of $X$. orientale subsp. italicum included the use of leaves by topical application as treatment of dermatitis, dermatophytosis, and as wounds cicatrized. In Turkey, the infusion of leaves and fruits of the same plant is used by native people as a diuretic, sedative, and antidepressant (Özdemir \& Alpinar 2015; Gürağaç Dereli et al. 2020). In China, the dried fruits of the genus species are used, often after heating to reduce its toxicity, as a component of traditional recipes with other herbs for the treatment of rhinitis (chronic or allergic), sinusitis, headache, hives, and arthritis (Su et al. 2016).

The other interesting case concerned Ampelodesmos mauritanicus. This plant, also called tenacious ampelodesme, is widespread in the North African region and dry regions from Greece to Spain. In the literature, the use of leaves of $A$. mauritanicus was reported in the construction of ancient dwellings, to bind the branches of vines in Italia (Salerno et al. 2005) or to manufacture brooms in North Africa (Cuénod et al. 1954). In our investigation, some of the informants indicated the use of $A$. mauritanicus as a vermifuge and anthelminthic. A variation on this treatment includes burning dried leaves in a container and then inhaling the emitted smoke, or placing the smoking ashes of the leaves in a room for 1 hour. In their review, Mohagheghzadeh et al. (2006) displayed the clinical efficacy of inhaling therapy for the treatment of pulmonary and neurological disorders essentially. They also explained this efficiency by the rapid delivery to the brain, large area of the pulmonary system, abundance of capillaries, and the thin air-blood barrier. When comparing our results with older studies, we found only two species used for the same purpose in similar ways: Artocarpus altilis (Parkinson) Fosb. from Tonga island (Whistler 1991) and Jasminum floribundum R. Br. ex Fresen. from Ethiopia (Mohagheghzadeh et al. 2006). Furthermore, no studies have been conducted to explore phytoconstituents and pharmacological activity of this plant. So, additional studies are needed to ensure the reliability of the data and scientifically prove the therapeutic effects of $A$. mauritanicus as mentioned by the informants.

Among the identified species, Marrubium alysson and Marrubium vulgare were designated by the same vernacular name (Table 2). This similarity of the vernacular names can be due either to the resemblance of the ports of the species (El Rhaffari \& Zaid 2002) or to the polymorphism which can characterize certain plants and consequently to the uses of the plant (Bahassan et al. 2014). Contrariwise, other plants, such as Anacyclus pyrethrum, Erigeron canadensis, Opuntia ficus-indica, Capparis spinosa, Fraxinus dimorpha, Cistanche phelypaea, Stipa tenacissima and Datura stramonium, recognize two vernacular names (Table 2). Still, according to Bahassan et al. (2014), this may explain the confusion regarding the identification of plants, the disease treated, or the multiplicity of plants used to treat the same pathology.

Of the recorded plants, it is worth noting the presence of 10 species appearing on the list of plant species protected by Executive Decree No. 12-03 of January 04, 2012 (JORADP 2012). They are Anvillea garcinii subsp radiata, Centaurea microcarpa, Juniperus communis, Juniperus oxycedrus, Juniperus phoenicea, Ephedra alata, Teucrium polium, Thymus satureioides, Fraxinus dimorpha, and Cedrus atlantica. It is also useful to note the presence of a significant number of toxic plants, of which the use should be limited to qualified persons (Table 2). The toxicity of a plant depends on several factors such as the part used, its method of administration, its concentration in active constituents, and the harvest season (Becker 1984; El Alami et al. 2016). These authors provided an example Artemisia herba alba, very toxic when it is harvested in summer but beneficial during all other seasons and Carlina gummifera, used in small doses but very toxic in large doses. 
Table 2. Spontaneous medicinal plants of the Aures region with families, voucher, local names, frequency of citation, part used, treated diseases (with ICPC-2 code), preparation and route of administration

\begin{tabular}{|c|c|c|c|c|c|c|}
\hline Family & $\begin{array}{l}\text { Scientific plant name (vouch- } \\
\text { ers) }\end{array}$ & Local name & $\begin{array}{l}\text { Frequency } \\
\text { citation }(\%)\end{array}$ & Part used & Treated diseases & Preparation \& administration \\
\hline Aizoaceae & $\begin{array}{l}\text { Mesembryanthemum nodiflo- } \\
\text { rum L. (Phb001) }\end{array}$ & Rahoul (A) & 4.03 & Aerial part & $\begin{array}{l}\text { General pain A01, wound } \\
\text { healing S18 }\end{array}$ & Cataplasm \\
\hline \multirow[t]{5}{*}{ Amaranthaceae } & $\begin{array}{l}\text { Anabasis articulata (Forssk.) } \\
\text { Moq. (PhB002) }\end{array}$ & Abelbal (C) & 3.02 & Aerial part & Diabetes T10, eczema S87 & $\begin{array}{l}\text { Decoction }(\mathrm{O}) \\
\text { Cataplasm }\end{array}$ \\
\hline & $\begin{array}{l}\text { Atriplex halimus } \mathbf{L} \text {. } \\
\text { (PhB003) }\end{array}$ & $\operatorname{Armi}(\mathrm{C})$ & 8.92 & Fresh leaves & $\begin{array}{l}\text { Diabetes T10, ovarian cyst } \\
\text { X99† } \\
\text { Cholesterol T79, rheumatic } \\
\text { pain L29, vomiting D10 } \\
\text { Rheumatic pain L29 }\end{array}$ & $\begin{array}{l}\text { Infusion }(\mathrm{O}) \\
\text { Decoction of powder }(\mathrm{O}) \\
\text { Cataplasm of powder mixed in olive }\end{array}$ \\
\hline & $\begin{array}{l}\text { Beta macrocarpa Guss. } \\
(\mathrm{PhB} 004)\end{array}$ & Aghzaz (C) & 2.85 & Leaves & $\begin{array}{l}\text { Anaemia B82 } \\
\text { Wound healing S18† }\end{array}$ & $\begin{array}{l}\text { Chewed } \\
\text { Cataplasm of powder mixed in olive } \\
\text { oil }\end{array}$ \\
\hline & $\begin{array}{l}\text { Caroxylon vermiculatum }(\mathrm{L} .) \\
\text { Akhani \& Roalson } \\
(\mathrm{PhB} 005)\end{array}$ & $\begin{array}{l}\text { Ghassoul } \\
\text { aachbi (A) }\end{array}$ & 2,18 & Fresh leaves & $\begin{array}{l}\text { Food poisoning A86†, stom- } \\
\text { achache D02 }\end{array}$ & Decoction $(\mathrm{O})$ \\
\hline & $\begin{array}{l}\text { Hammada scoparia (Pomel) Iljin } \\
\text { (PhB006) }\end{array}$ & Remth (A) & 2.01 & Leaves & $\begin{array}{l}\text { Diabetes T10 } \\
\text { Wound healing S18 }\end{array}$ & $\begin{array}{l}\text { Decoction }(\mathrm{O}) \\
\text { Cataplasm of ash mixed in olive oil }\end{array}$ \\
\hline \multirow[t]{2}{*}{ Anacardiaceae } & $\begin{array}{l}\text { Pistacia lentiscus L. } \\
\text { (PhB007) }\end{array}$ & Thiddekth (C) & 23.69 & Resin & $\begin{array}{l}\text { Respiratory system pain R01, } \\
\text { wound healing S18 } \\
\text { Gastric ulcer D } 86 \text {, gum care } \\
\text { D19 }\end{array}$ & $\begin{array}{l}\text { Friction with extracted oil } \\
\text { Chewing }\end{array}$ \\
\hline & $\begin{array}{l}\text { Searsia tripartita (Ucria) Mof- } \\
\text { fett }(\mathrm{PhB} 008)\end{array}$ & Lok (A) & 5.21 & $\begin{array}{l}\text { Bark } \\
\text { Fruits }\end{array}$ & $\begin{array}{l}\text { Mouth ulcers D20† } \\
\text { Diarrhoea D11 }\end{array}$ & $\begin{array}{l}\text { Powder (MW) } \\
\text { Maceration in milk (O) }\end{array}$ \\
\hline \multirow[t]{4}{*}{ Apiaceae } & $\begin{array}{l}\text { Daucus carota } \mathrm{L} . \\
(\mathrm{PhB} 009)\end{array}$ & Afasnoukh (C) & 9.24 & $\begin{array}{l}\text { Seeds } \\
\text { Tubers }\end{array}$ & $\begin{array}{l}\text { Irregular menstruation X07 } \\
\text { Constipation D12 }\end{array}$ & $\begin{array}{l}\text { Infusion }(\mathrm{O}) \\
\text { Fresh Juice }(\mathrm{O})\end{array}$ \\
\hline & Ferula communis L. $\$(\mathrm{PhB} 010)$ & Ouffal (C) & 3.36 & $\begin{array}{l}\text { Fruits } \\
\text { Latex }\end{array}$ & $\begin{array}{l}\text { Headache N01 } \\
\text { Tonsilitis R76†, haemorrhage } \\
\text { A10 }\end{array}$ & $\begin{array}{l}\text { Decoction }(\mathrm{O}) \\
\text { Cataplasm }\end{array}$ \\
\hline & $\begin{array}{l}\text { Ridolfia segetum (Guss.) Moris } \\
\text { (PhB011) }\end{array}$ & $\begin{array}{l}\text { El karwiya el } \\
\text { aamya }(\mathrm{A})\end{array}$ & 2.85 & Seeds & $\begin{array}{l}\text { Constipation D12, flatulence } \\
\text { D08, irregular menstruation } \\
\text { X07, lactation insufficiency } \\
\text { W19 }\end{array}$ & Infusion $(\mathrm{O})$ \\
\hline & Thapsia garganica $\mathrm{L} . \$(\mathrm{PhB} 012)$ & Aderiass $(\mathrm{C})$ & 9.24 & $\begin{array}{l}\text { Leaves } \\
\text { Bulbs }\end{array}$ & $\begin{array}{l}\text { Rheumatic pain L29, weakness } \\
\text { A04 } \\
\text { Arthritis L20 }\end{array}$ & $\begin{array}{l}\text { Decoction }(\mathrm{O}) \\
\text { Cataplasm of bulb macerated in oil }\end{array}$ \\
\hline
\end{tabular}




\begin{tabular}{|c|c|c|c|c|c|c|}
\hline Apocynaceae & $\begin{array}{l}\text { Nerium oleander L.F } \\
(\mathrm{PhB} 013)\end{array}$ & Tlili (C) & 6.05 & Fresh leaves & $\begin{array}{l}\text { Hair care S24 } \\
\text { Diabetic foot ulcer T89 } \\
\text { Post-partum loss of vaginal } \\
\text { flexibility } \mathrm{W} 18 \dagger\end{array}$ & $\begin{array}{l}\text { Decoction (HW) } \\
\text { Cataplasm } \\
\text { Steam of boiled leaves }\end{array}$ \\
\hline & $\begin{array}{l}\text { Pergularia tomentosa L. } \\
(\mathrm{PhB} 014)\end{array}$ & Ghalga (A) & 6.21 & Latex & $\begin{array}{l}\text { Arthritis L20, corn S20, } \\
\text { epidermal cyst S19, snake and } \\
\text { scorpion bite S13, wart S03 }\end{array}$ & Cataplasm \\
\hline Asparagaceae & $\begin{array}{l}\text { Agave americana } \mathrm{L} . \\
(\mathrm{PhB} 015)\end{array}$ & Sabar (A) & 8.23 & Aerial parts & $\begin{array}{l}\text { Gastric ulcer D86, hepatotox- } \\
\text { icity D99 } \\
\text { Skin inflammation S01, skin } \\
\text { softener S21, wound healing } \\
\text { S18 }\end{array}$ & $\begin{array}{l}\text { Fresh Juice }(\mathrm{O}) \\
\text { Cataplasm }\end{array}$ \\
\hline \multirow[t]{8}{*}{ Asteraceae } & $\begin{array}{l}\text { Anacyclus clavatus (Desf.) Pers. } \\
\text { (PhB016) }\end{array}$ & Babounj (A) & 7.05 & Aerial parts & Gastric ulcer D86, fever A03 $†$ & Infusion/decoction (O) \\
\hline & $\begin{array}{l}\text { Anacyclus pyrethrum (L.) Lag.\$ } \\
\text { (PhB017) }\end{array}$ & $\begin{array}{l}\text { Agargarha, } \\
\text { Tigenthast (C) }\end{array}$ & 7.56 & Roots & Infertility W15 & $\begin{array}{l}\text { Decoction mixed in honey with } \\
\text { ginger and onion }(\mathrm{O})\end{array}$ \\
\hline & & & & & Tonsilitis R76, tooth pain D19 & Decoction (MW) \\
\hline & $\begin{array}{l}\text { Anvillea garcinii subsp. radiata } \\
\text { (Coss. \& Durieu) Anderb. } \\
\text { (PhB018) }\end{array}$ & Nugd (A) & 6.05 & $\begin{array}{l}\text { Seeds } \\
\text { Fresh leaves }\end{array}$ & $\begin{array}{l}\text { Dyspepsy D07 } \\
\text { Influenza R80 } \\
\text { Low back complaint L03 }\end{array}$ & $\begin{array}{l}\text { Infusion }(\mathrm{O}) \\
\text { Infused honey }(\mathrm{O}) \\
\text { Suppository made with powder and } \\
\text { butter }(\mathrm{R})\end{array}$ \\
\hline & $\begin{array}{l}\text { Artemisia absinthium L.+ } \\
(\mathrm{PhB019)}\end{array}$ & $\begin{array}{l}\text { Thasata } \mathrm{n} \\
\text { Meriem }(\mathrm{C})\end{array}$ & 9.07 & $\begin{array}{l}\text { Aerial parts } \\
\text { Inflores- } \\
\text { cence }\end{array}$ & $\begin{array}{l}\text { Dyspepsy D07, loss of appetite } \\
\text { T03, stomachache D02, weak- } \\
\text { ness A04, worms D96 } \\
\text { Bruise/Contusion S16, insect } \\
\text { bite S12 }\end{array}$ & $\begin{array}{l}\text { Infusion of powder mixed with } \\
\text { liquorice and anise }(\mathrm{O}) \\
\text { Decoction }(\mathrm{T})\end{array}$ \\
\hline & $\begin{array}{l}\text { Artemisia campestris L. } \\
(\mathrm{PhB} 020)\end{array}$ & Thgouft (C) & 20.50 & $\begin{array}{l}\text { Inflores- } \\
\text { cence }\end{array}$ & $\begin{array}{l}\text { Convulsion N07, dyspepsy } \\
\text { D07, vomiting D11, menstrual } \\
\text { pain X02 } \\
\text { Diabetes T10 }\end{array}$ & $\begin{array}{l}\text { Infusion }(\mathrm{O}) \\
\text { Decoction }(\mathrm{O})\end{array}$ \\
\hline & $\begin{array}{l}\text { Artemisia herba-alba Asso } \\
(\mathrm{PhB} 021)\end{array}$ & Tzri (C) & 19.83 & $\frac{\text { Roots }}{\text { Aerial parts }}$ & $\begin{array}{l}\text { Menstrual pain X02, stomach- } \\
\text { ache D02, vomiting D11 } \\
\text { Tooth pain D19 } \\
\text { Food poisoning A86 } \\
\text { Rheumatic pain L29 }\end{array}$ & $\begin{array}{l}\text { Infusion }(\mathrm{O}) \\
\text { Decoction (MW) } \\
\text { Decoction mixed with milk }(\mathrm{O}) \\
\text { Cataplasm of powder mixed with } \\
\text { thyme and lavender }\end{array}$ \\
\hline & $\begin{array}{l}\text { Brocchia cinerea (Delile) Vis. } \\
(\mathrm{PhB} 022)\end{array}$ & $\begin{array}{l}\text { Kerchet } \\
\text { lekhrouf (A) }\end{array}$ & 5.37 & Aerial parts & $\begin{array}{l}\text { Abdominal colic D01, chills } \\
\text { A02, cough R05, dyspepsy } \\
\text { D07 }\end{array}$ & Infusion (O) \\
\hline
\end{tabular}




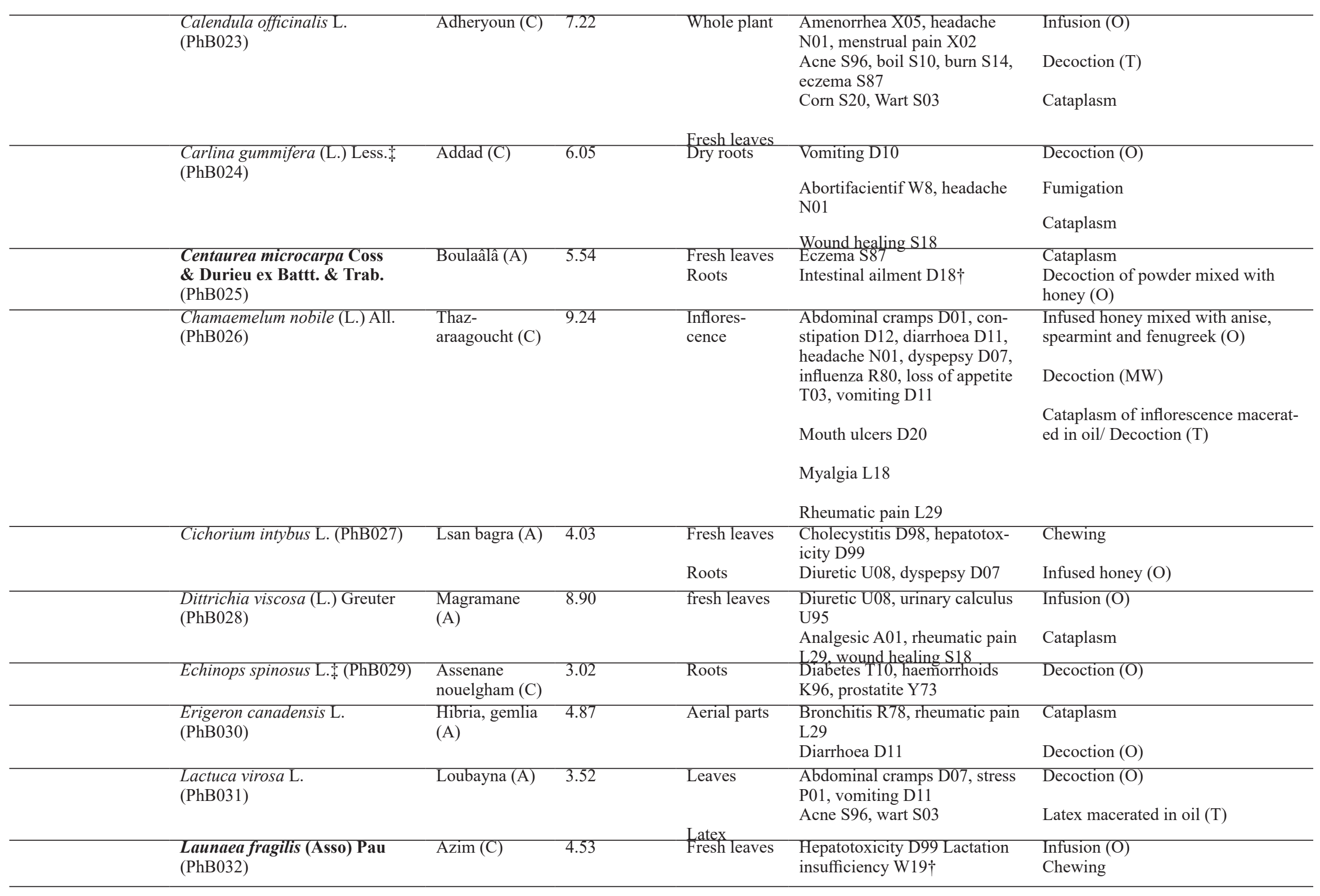




\begin{tabular}{|c|c|c|c|c|c|c|}
\hline & $\begin{array}{l}\text { Pallenis spinosa (L.) Cass. } \\
(\mathrm{PhB} 033)\end{array}$ & Rebian (A) & 1.68 & Aerial parts & $\begin{array}{l}\text { Diabetes T10, stomachache } \\
\text { D02† }\end{array}$ & Infusion/decoction (O) \\
\hline & $\begin{array}{l}\text { Pulicaria vulgaris Gaertn. } \\
(\mathrm{PhB} 034)\end{array}$ & Sefçac (A) & 2.18 & Aerial parts & $\begin{array}{l}\text { Stomachache D02 } \uparrow \text {, tooth pain } \\
\text { D19† }\end{array}$ & Decoction (O) \\
\hline & $\begin{array}{l}\text { Scolymus hispanicus L. } \\
(\mathrm{PhB} 035)\end{array}$ & Scolime (A) & 3.19 & Leaves & Stomachache D02 & Infusion (O) \\
\hline & $\begin{array}{l}\text { Senecio vulgaris } \mathrm{L} . \\
(\mathrm{PhB} 036)\end{array}$ & Chikh rabii $(\mathrm{A})$ & 3.69 & $\begin{array}{l}\text { Inflores- } \\
\text { cence }\end{array}$ & $\begin{array}{l}\text { Amenorrhea X05, menstrual } \\
\text { pain X02, rheumatic pain L29, } \\
\text { varicose K95† }\end{array}$ & Decoction $(\mathrm{O})$ \\
\hline & $\begin{array}{l}\text { Silybum marianum (L.) Gaertn. } \\
\text { (PhB037) }\end{array}$ & $\begin{array}{l}\text { Thanwarth } \\
\text { noussenane } \\
\text { (C) }\end{array}$ & 3.69 & Roots & $\begin{array}{l}\text { Jaundice D13, liver disease } \\
\text { D97 }\end{array}$ & Decoction $(\mathrm{O})$ \\
\hline & Sonchus oleraceus L. (PhB038) & Tilfef $(\mathrm{A})$ & 2.52 & $\begin{array}{l}\text { Fresh leaves, } \\
\text { dry roots, } \\
\text { Juice }\end{array}$ & $\begin{array}{l}\text { Cholesterol T79, stomachache } \\
\text { D02 } \\
\text { Wart S03 }\end{array}$ & $\begin{array}{l}\text { Infusion/decoction }(\mathrm{O}) \\
\text { Cataplasm }\end{array}$ \\
\hline & $\begin{array}{l}\text { Taraxacum officinale F.H. Wigg. } \\
\text { (PhB039) }\end{array}$ & Thalma (C) & 8.06 & Leaves & $\begin{array}{l}\text { Diuretic U08, loss of appetite } \\
\text { T03 }\end{array}$ & Infusion (O) \\
\hline & $\begin{array}{l}\text { Xanthium orientale subsp. } \\
\text { italicum (Moretti) Greuter* } \\
\text { (PhB040) }\end{array}$ & Habala (A) & 1.51 & Leaves & $\begin{array}{l}\text { Dermatistis S } 87 \dagger \text {, dermato- } \\
\text { phytosis S74 } \uparrow \text {, wound healing } \\
\text { S18† }\end{array}$ & Cataplasm \\
\hline \multirow[t]{3}{*}{ Brassicaceae } & $\begin{array}{l}\text { Capsella bursa-pastoris (L.) } \\
\text { Medik. (PhB041) }\end{array}$ & Kis er-raii (A) & 5.04 & Aerial parts & $\begin{array}{l}\text { Haemorrhoids K96, hot flashes } \\
\text { X11, menstrual bleeding X08, } \\
\text { varicose K95 }\end{array}$ & Decoction $(\mathrm{O})$ \\
\hline & & & & & Wound healing S18 & Tincture $(\mathrm{T})$ \\
\hline & $\begin{array}{l}\text { Sinapis arvensis L. } \\
(\mathrm{PhB} 042)\end{array}$ & Khardel (A) & 4.03 & Fresh leaves & Dyspepsy D07 & Eaten raw or cooked \\
\hline Boraginaceae & Borago officinalis L. (PhB043) & Lsan thawr $(\mathrm{A})$ & 7.05 & $\begin{array}{l}\text { Inflores- } \\
\text { cence } \\
\text { Juice } \\
\text { Leaves }\end{array}$ & $\begin{array}{l}\text { Diuretic U08, hyperhidrosis } \\
\text { A09 } \\
\text { Diuretic U08 } \\
\text { Skin inflammation S01 }\end{array}$ & $\begin{array}{l}\text { Infusion }(\mathrm{O}) \\
\text { Fresh juice }(\mathrm{O}) \\
\text { Cataplasm }\end{array}$ \\
\hline Cactaceae & $\begin{array}{l}\text { Opuntia ficus-indica (L.) Mill. } \\
\text { (PhB044) }\end{array}$ & $\begin{array}{l}\text { Aaroug (C) } \\
\text { Troumoucht } \\
\text { (C) Taker- } \\
\text { moust (C) }\end{array}$ & 8.23 & $\begin{array}{l}\text { Leaves } \\
\text { Fruits }\end{array}$ & $\begin{array}{l}\text { Boil S10 } \\
\text { Diarrhoea D11, flatulence D08 }\end{array}$ & $\begin{array}{l}\text { Cataplasm } \\
\text { Eaten raw }\end{array}$ \\
\hline Capparaceae & $\begin{array}{l}\text { Capparis spinosa L. } \\
(\mathrm{PhB} 045)\end{array}$ & $\begin{array}{l}\text { Kabbar (A) } \\
\text { Tiloulout (C) }\end{array}$ & 9.07 & $\begin{array}{l}\text { Leaves, } \\
\text { Roots bark }\end{array}$ & $\begin{array}{l}\text { Stomachache D02 } \\
\text { Haemorrhage A10, loss of } \\
\text { appetite T03, vaginal infection } \\
\text { X92 }\end{array}$ & $\begin{array}{l}\text { Crushed and macerated in vinegare } \\
\text { (O) } \\
\text { Decoction }(\mathrm{O})\end{array}$ \\
\hline Cleomaceae & $\begin{array}{l}\text { Cleome arabica L. } \\
(\mathrm{PhB} 046)\end{array}$ & Netteina (A) & 8.73 & Leaves & $\begin{array}{l}\text { Rheumatic pain L29, wound } \\
\text { healing S18 }\end{array}$ & $\begin{array}{l}\text { Cataplasm of powder mixed in olive } \\
\text { oil (T) }\end{array}$ \\
\hline
\end{tabular}




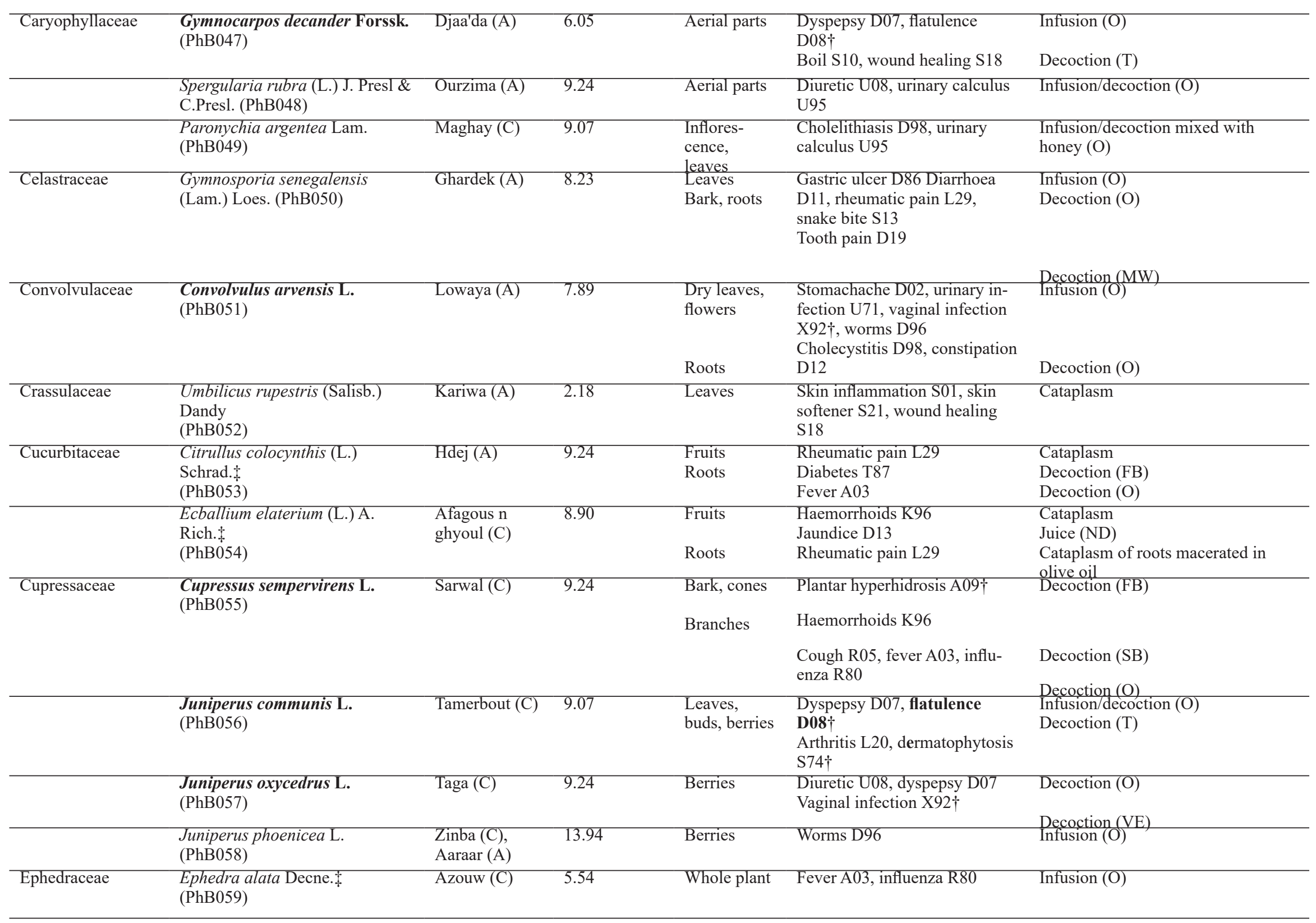




\begin{tabular}{|c|c|c|c|c|c|c|}
\hline Euphorbiaceae & $\begin{array}{l}\text { Euphorbia helioscopia L. } \\
(\mathrm{PhB060)}\end{array}$ & Hanghouth (C) & 8.06 & $\begin{array}{l}\text { Leaves } \\
\text { Latex }\end{array}$ & $\begin{array}{l}\text { Snake and scorpion bite S13† } \\
\text { Corn S20, wart S03 }\end{array}$ & $\begin{array}{l}\text { Cataplasm } \\
\text { Cataplasm }\end{array}$ \\
\hline & Ricinus communis L.† (PhB061) & Kherwaa (A) & 8.23 & $\begin{array}{l}\text { Fresh leaves } \\
\text { Seeds }\end{array}$ & $\begin{array}{l}\text { Fever A03 } \\
\text { Skin softener S21 }\end{array}$ & $\begin{array}{l}\text { Cataplasm } \\
\text { Cataplasm of extracted oil }\end{array}$ \\
\hline \multirow[t]{5}{*}{ Fabaceae } & $\begin{array}{l}\text { Astragalus armatus Willd. } \\
\text { (PhB062) }\end{array}$ & $\begin{array}{l}\text { Hawejmayath } \\
\text { (C) }\end{array}$ & 6.05 & Aerial parts & $\begin{array}{l}\text { Lactation insufficiency W19† } \\
\text { Diabetes T10 }\end{array}$ & Infusion $(\mathrm{O})$ \\
\hline & $\begin{array}{l}\text { Ceratonia siliqua L. } \\
(\mathrm{PhB} 063)\end{array}$ & Thisliwgha (C) & 5.21 & $\begin{array}{l}\text { Roots } \\
\text { Flowers } \\
\text { Fruits }\end{array}$ & $\begin{array}{l}\text { Skin softener S21 } \\
\text { Diarrhoea D11 }\end{array}$ & $\begin{array}{l}\text { Decoction }(\mathrm{O}) \\
\text { Cataplasm } \\
\text { Chewing }\end{array}$ \\
\hline & Cytisus villosus Pourr. (PhB064) & Hiloukath (C) & 4.70 & Aerial parts & Wound healing S18 & Cataplasm \\
\hline & $\begin{array}{l}\text { Medicago sativa } \mathrm{L} . \\
(\mathrm{PhB} 065)\end{array}$ & Safsfa (A) & 7.05 & Leaves & Diabetes T10 & Infusion $(\mathrm{O})$ \\
\hline & $\begin{array}{l}\text { Retama raetam (Forssk.) Webb } \\
\text { \& Berthel. }(\mathrm{PhB} 066)\end{array}$ & Rtem (A) & 4.87 & Aerial parts & $\begin{array}{l}\text { Rheumatic pain L29, snake and } \\
\text { scorpion bite S13 }\end{array}$ & Cataplasm \\
\hline Fagaceae & Quercus ilex L. (PhB067) & $\begin{array}{l}\text { Akkerouche } \\
\text { (C) }\end{array}$ & 8.57 & $\begin{array}{l}\text { Fruits } \\
\text { Bark }\end{array}$ & $\begin{array}{l}\text { Diarrhoea D11 } \\
\text { Tooth pain D19 } \\
\text { Chapped skin S17 } \\
\text { Conjunctivitis F70 }\end{array}$ & $\begin{array}{l}\text { Eaten raw } \\
\text { Decoction (MW) } \\
\text { Decoction (FB) } \\
\text { Decoction (EC) }\end{array}$ \\
\hline \multirow[t]{4}{*}{ Lamiaceae } & $\begin{array}{l}\text { Ajuga iva (L.) Schreb. } t \\
\text { (PhB068) }\end{array}$ & Tchangoura (C) & 9.24 & Aerial parts & $\begin{array}{l}\text { Abdominal pain D01, cho- } \\
\text { lecystitis D98, fever A03, } \\
\text { induction of labor W29†, } \\
\text { vomiting D11 Rheumatic pain } \\
\text { L29, wound healing S18 }\end{array}$ & $\begin{array}{l}\text { Decoction }(\mathrm{O}) \\
\text { Cataplasm }\end{array}$ \\
\hline & $\begin{array}{l}\text { Marrubium alysson L. } \\
\text { (PhB069) }\end{array}$ & Merriweth (C) & 9.24 & Aerial parts & $\begin{array}{l}\text { Asthma R96, cough R05 } \\
\text { Boil S10†, fever A03 }\end{array}$ & $\begin{array}{l}\text { Decoction mixed with honey }(\mathrm{O}) \\
\text { Cataplasm }\end{array}$ \\
\hline & $\begin{array}{l}\text { Marrubium vulgare L. } \\
(\mathrm{PhB} 070)\end{array}$ & Merriweth (C) & 21.69 & Aerial parts & $\begin{array}{l}\text { Anaemia B82 } \dagger \text {, dyspepsy D07 } \\
\text { Bronchitis R78 } \\
\text { Cough R05 } \\
\text { Respiratory disorder R01 } \\
\text { Bronchitis R78, fever A03† }\end{array}$ & $\begin{array}{l}\text { Infused honey } \\
\text { Infusion mixed with honey and } \\
\text { plantain } \\
\text { Infusion mixed with honey and blue } \\
\text { gum (O) } \\
\text { Infusion (ND) } \\
\text { Cataplasm }\end{array}$ \\
\hline & $\begin{array}{l}\text { Rosmarinus officinalis } \mathrm{L} \text {. } \\
(\mathrm{PhB} 071)\end{array}$ & Aziir (C) & 24.20 & Flowers & $\begin{array}{l}\text { Irregular menstruationX07†, } \\
\text { stomachache D02 } \\
\text { Flatulence D08 } \\
\text { Burn S14, wound cleansing } \\
\text { S18 } \\
\text { Myalgia L18, rheumatic pain } \\
\text { L29 }\end{array}$ & $\begin{array}{l}\text { Infusion/decoction }(\mathrm{O}) \\
\text { Infused honey }(\mathrm{O}) \\
\text { Decoction }(\mathrm{T}) \\
\text { Tincture/Cataplasm }\end{array}$ \\
\hline
\end{tabular}




\begin{tabular}{|c|c|c|c|c|c|c|}
\hline & $\begin{array}{l}\text { Teucrium polium L. } \\
\text { (PhB072) }\end{array}$ & Khayata (A) & 5.21 & Aerial parts & $\begin{array}{l}\text { Gastric ulcer D86 } \\
\text { Stomachache D02 } \\
\text { Wound healing S18 }\end{array}$ & $\begin{array}{l}\text { Infusion }(\mathrm{O}) \\
\text { Decoction }(\mathrm{O}) \\
\text { Cataplasm }\end{array}$ \\
\hline & $\begin{array}{l}\text { Thymus saturejoides Coss. } \\
\text { (PhB073) }\end{array}$ & Ziitra (A) & 9.07 & Aerial parts & $\begin{array}{l}\text { Influenza R80, stomachache } \\
\text { D02 }\end{array}$ & Infusion/decoction (O) \\
\hline & Vitex agnus-castus L. (PhB074) & $\begin{array}{l}\text { Kaf Meriem } \\
\text { (A) }\end{array}$ & 8.73 & Seeds & $\begin{array}{l}\text { Lactation insufficiency W19, } \\
\text { menstrual pain X02 } \\
\text { Urinary calculus U95 }\end{array}$ & Infusion/decoction (O) \\
\hline \multirow[t]{2}{*}{ Malvaceae } & $\begin{array}{l}\text { Alcea rosea L. } \\
\text { (PhB075) }\end{array}$ & Khobeiza (A) & 2.85 & $\begin{array}{l}\text { Leaves, } \\
\text { flowers }\end{array}$ & $\begin{array}{l}\text { Bronchitis R78, constipation } \\
\text { D12 }\end{array}$ & Infusion with liquorice $(\mathrm{O})$ \\
\hline & $\begin{array}{l}\text { Malva sylvestris L. } \\
\text { (PhB076) }\end{array}$ & Amejjir (C) & 6.55 & $\begin{array}{l}\text { Leaves, } \\
\text { flowers }\end{array}$ & $\begin{array}{l}\text { Constipation D12 } \\
\text { Respiratory disorders R01 } \\
\text { Low back pain L03 } \\
\text { Mouth ulcers D20 } \\
\text { Eye inflammation F73 }\end{array}$ & $\begin{array}{l}\text { Chewing } \\
\text { Infusion/decoction (O) } \\
\text { Decoction (T) } \\
\text { Decoction (MW) } \\
\text { Decoction (ED) }\end{array}$ \\
\hline Meliaceae & $\begin{array}{l}\text { Azadirachta indica A. Juss. } \\
\text { (PhB077) }\end{array}$ & $\operatorname{Nim}(\mathrm{A})$ & 3.08 & Bark & $\begin{array}{l}\text { Scorpion bite S13, wound } \\
\text { cleansing S18 }\end{array}$ & Decoction $(\mathrm{T})$ \\
\hline \multirow[t]{2}{*}{ Myrtaceae } & $\begin{array}{l}\text { Eucalyptus globulus Labill.\$ } \\
\text { (PhB078) }\end{array}$ & Kalitousse (A) & 9.24 & Leaves & $\begin{array}{l}\text { Diabetes T10 } \\
\text { Allergic rhinitis R97, asthma } \\
\text { R96, influenza R80, insect bite } \\
\text { S12 }\end{array}$ & $\begin{array}{l}\text { Decoction (O) } \\
\text { Fumigation (I) }\end{array}$ \\
\hline & $\begin{array}{l}\text { Myrtus communis L. } \\
\text { (PhB079) }\end{array}$ & Rihan (A) & 8.40 & $\begin{array}{l}\text { Leaves } \\
\text { Eruits }\end{array}$ & $\begin{array}{l}\text { Dyspepsy D07, respiratory } \\
\text { disorders R01 } \\
\text { Diarrhoea D11 }\end{array}$ & $\begin{array}{l}\text { Infusion with rosemary and } \\
\text { fenugreek }(\mathrm{O}) \\
\text { Chewing }\end{array}$ \\
\hline Nitrariaceae & Peganum harmala L.t (PhB080) & Harmel $(A)$ & 8.74 & $\begin{array}{l}\text { Aerial parts } \\
\text { Leaves }\end{array}$ & $\begin{array}{l}\text { Gingivitis D19 } \\
\text { Hair care S24 } \\
\text { Arthritis L20 }\end{array}$ & $\begin{array}{l}\text { Decoction (MW) } \\
\text { Decoction (HW) } \\
\text { Cataplasm }\end{array}$ \\
\hline Oleaceae & $\begin{array}{l}\text { Fraxinus dimorpha Coss. \& } \\
\text { Durieu (PhB081) }\end{array}$ & $\begin{array}{l}\text { Thouzzalt (C) } \\
\text { N'chem (C) }\end{array}$ & 1.17 & Branches & Fever A03 & Decoction $(\mathrm{O})$ \\
\hline Orobanchaceae & $\begin{array}{l}\text { Cistanche phelypaea (L.) Cout. } \\
(\mathrm{PhB} 082)\end{array}$ & $\begin{array}{l}\text { Dhanoun (A) } \\
\text { Djaafil (A) }\end{array}$ & 8.23 & Roots & $\begin{array}{l}\text { Diabetes T10, intestinal pain } \\
\text { D18 }\end{array}$ & Decoction $(\mathrm{O})$ \\
\hline Oxalidaceae & Oxalis pes-caprae L. (PhB083) & Quoriça $(A)$ & 2.18 & Fresh leaves & Mouth ulcer D20 & Chewing \\
\hline Papaveraceae & $\begin{array}{l}\text { Papaver rhoeas L. } \\
\text { (PhB084) }\end{array}$ & Thakouche (C) & 4.36 & Flowers & $\begin{array}{l}\text { Bronchitis R78, stress P01, } \\
\text { tonsilitis R76 } \\
\text { Eye inflammation F73 }\end{array}$ & $\begin{array}{l}\text { Infusion }(\mathrm{O}) \\
\text { Infusion (ED) }\end{array}$ \\
\hline \multirow[t]{4}{*}{ Pinaceae } & $\begin{array}{l}\text { Cedrus atlantica (Endl.) Manetti } \\
\text { ex Carrière }+(\mathrm{PhB} 085)\end{array}$ & Ad'il (C) & 7.89 & Bark & $\begin{array}{l}\text { Liver disease D97, hyperhidro- } \\
\text { sis A09 }\end{array}$ & Decoction $(\mathrm{O})$ \\
\hline & Pinus halepensis Mill. (PhB086) & Snobar (A) & 20.84 & Buds & $\begin{array}{l}\text { Respiratory disorder R01, } \\
\text { urinary infection U71 }\end{array}$ & Infusion (O) \\
\hline & & & & Bark & $\begin{array}{l}\text { Expectoration R25, rheumatic } \\
\text { pain L29 }\end{array}$ & Ointment $(\mathrm{T})$ \\
\hline & & & & Resin & $\begin{array}{l}\text { Cough R05, gastric ulcer D } 86 \text {, } \\
\text { heartburn D03, nausea D09 }\end{array}$ & Chewed mixed with honey $(\mathrm{O})$ \\
\hline
\end{tabular}




\begin{tabular}{|c|c|c|c|c|c|c|}
\hline Plantaginaceae & Globularia alypum L.\$ (PhB087) & Tasselgha (C) & 7.39 & Whole plant & $\begin{array}{l}\text { Fever A03, stomachache D02, } \\
\text { weakness A04 }\end{array}$ & Infusion $(\mathrm{O})$ \\
\hline & $\begin{array}{l}\text { Plantago major L. } \\
\text { (PhB088) }\end{array}$ & $\begin{array}{l}\text { Lsan el-hamel } \\
\text { lekbir (A) }\end{array}$ & 7.73 & Leaves & $\begin{array}{l}\text { Diarrhoea D11 } \\
\text { Asthma R96 } \\
\text { Conjunctivis } \\
\text { Tonsilitis R76 } \\
\text { Haemorrhoids K96 } \\
\text { Boil S10 }\end{array}$ & $\begin{array}{l}\text { Infusion (O) } \\
\text { Infusion with white horehound (O) } \\
\text { Decoction (ED) } \\
\text { Decoction (MW) } \\
\text { Decoction (SB) } \\
\text { Cataplasm }\end{array}$ \\
\hline Plumbaginaceae & $\begin{array}{l}\text { Limoniastrum guyonianum } \\
\text { Boiss. } \\
(\mathrm{PhB} 089)\end{array}$ & Zeïta (A) & 8.23 & Aerial parts & Bronchitis R78, tonsilitis R76 & Decoction $(\mathrm{O})$ \\
\hline \multirow[t]{5}{*}{ Poaceae } & $\begin{array}{l}\text { Ampelodesmos mauritanicus } \\
\text { (Poir.) T. Durand \& Schinz* } \\
(\mathrm{PhB} 090)\end{array}$ & Ideles (C) & 5.54 & Leaves & Worms D96† & Fumigation (I) \\
\hline & Avena sterilis L. (PhB091) & Akhertal (C) & 1.01 & Seeds & Diabetes T10, weakness A04 & Eaten cooked bread \\
\hline & $\begin{array}{l}\text { Cynodon dactylon (L.) Pers. } \\
\text { (PhB092) }\end{array}$ & Njem (A) & 4.87 & Rhizome & $\begin{array}{l}\text { Arthritis L20, rheumatic pain } \\
\text { L29, urinary infection U71 }\end{array}$ & Decoction (O) \\
\hline & $\begin{array}{l}\text { Macrochloa tenacissima (L.) } \\
\text { Kunth }(\mathrm{PhB} 093)\end{array}$ & $\begin{array}{l}\text { Ikelzi, as- } \\
\text { senagh (C) }\end{array}$ & 6.21 & Leaves & $\begin{array}{l}\text { Hypertension K86, stomach- } \\
\text { ache D } 02 \uparrow\end{array}$ & Decoction $(\mathrm{O})$ \\
\hline & $\begin{array}{l}\text { Stipagrostis pungens (Desf.) } \\
\text { De Winter subsp. Pungens } \\
\text { (PhB094) }\end{array}$ & Drin $(\mathrm{A})$ & 6.21 & Aerial parts & $\begin{array}{l}\text { Constipation D12, stomach- } \\
\text { ache D02, urinary calculus } \\
\text { U95† }\end{array}$ & Infusion/decoction (O) \\
\hline \multirow[t]{2}{*}{ Polygonaceae } & $\begin{array}{l}\text { Polygonum aviculare L. } \\
\text { (PhB095) }\end{array}$ & $\begin{array}{l}\text { Taam elfarkh } \\
\text { (A) }\end{array}$ & 5.21 & Aerial parts & $\begin{array}{l}\text { Diarrhoea D11, urinary cal- } \\
\text { culus U95 Haemorrhage A10, } \\
\text { wound healing S18 }\end{array}$ & $\begin{array}{l}\text { Infusion (O) } \\
\text { Cataplasm }\end{array}$ \\
\hline & $\begin{array}{l}\text { Rumex acetosa L. } \\
\text { (PhB096) }\end{array}$ & $\begin{array}{l}\text { Semmamout } \\
\text { (C) }\end{array}$ & 3.52 & Leaves & Boil S10 & Cataplasm of cooked leaves \\
\hline Portulacaceae & Portulaca oleracea L. (PhB097) & Berzgala $(\mathrm{A})$ & 2.01 & Aerial parts & Diuretic U08, Dyspepsy D07 & $\begin{array}{l}\text { Juice }(\mathrm{O}) \\
\text { Chewing }\end{array}$ \\
\hline Ranunculaceae & Adonis dentata Delile (PhB098) & $\begin{array}{l}\text { Ain el hedjla } \\
\text { (A) }\end{array}$ & 2.68 & $\begin{array}{l}\text { Inflores- } \\
\text { cence }\end{array}$ & Cardiac stimulant K05 & Infusion of powder $(\mathrm{O})$ \\
\hline Resedaceae & $\begin{array}{l}\text { Reseda lutea L. } \\
\text { (PhB099) }\end{array}$ & $\begin{array}{l}\text { Djeneb } \\
\text { lakhrouf (A) }\end{array}$ & 6.55 & $\begin{array}{l}\text { Fresh leaves } \\
\text { Inflores- } \\
\text { cence }\end{array}$ & $\begin{array}{l}\text { Diuretic U08 } \\
\text { Liver disease D97 }\end{array}$ & $\begin{array}{l}\text { Chewed } \\
\text { Infusion }(\mathrm{O})\end{array}$ \\
\hline \multirow[t]{2}{*}{ Rhamnaceae } & $\begin{array}{l}\text { Rhamnus alaternus L. } \\
(\mathrm{PhB} 100)\end{array}$ & Amlilis (C) & 7.89 & Leaves & $\begin{array}{l}\text { Haemorrhoids K96 } \dagger \text {, stom- } \\
\text { achache D02, urinary calculus } \\
\text { U95 }\end{array}$ & Infusion (O) \\
\hline & $\begin{array}{l}\text { Ziziphus lotus (L.) Lam. } \\
\text { (PhB101) }\end{array}$ & Thazzgarth (C) & 7.73 & Fruits & Urinary calculus U95 & $\begin{array}{l}\text { Decoction mixed with honey and } \\
\text { Algerian tea }(\mathrm{O})\end{array}$ \\
\hline
\end{tabular}




\begin{tabular}{|c|c|c|c|c|c|c|}
\hline Rosaceae & $\begin{array}{l}\text { Crataegus laevigata (Poir.) DC. } \\
\text { (PhB102) }\end{array}$ & $\begin{array}{l}\text { Thazaarourth } \\
\text { (C) }\end{array}$ & 8.40 & Flowers & $\begin{array}{l}\text { Hypertension K86, urinary } \\
\text { calculus U95 }\end{array}$ & $\begin{array}{l}\text { Decoction of powder mixed with } \\
\text { honey }(O)\end{array}$ \\
\hline & $\begin{array}{l}\text { Rosa canina } \mathrm{L} . \\
\text { (PhB103) }\end{array}$ & Achdrit (C) & 5.04 & Fruits & $\begin{array}{l}\text { Stomachache D02, urinary } \\
\text { infection U71 }\end{array}$ & Decoction $(\mathrm{O})$ \\
\hline & & & & & Eye inflammation F73 & Decoction (EC) \\
\hline & $\begin{array}{l}\text { Rubus ulmifolius Schott } \\
\text { (PhB104) }\end{array}$ & Kilssa (C) & 7.39 & Fuits & $\begin{array}{l}\text { Anaemia B82 } \\
\text { Pregnancy pain W29† }\end{array}$ & Infusion (O) \\
\hline \multirow[t]{2}{*}{ Rutaceae } & Ruta montana (L.) L.† (PhB105) & Aourmi (C) & 6.38 & Aerial parts & Head lice S72 & Decoction (HW) \\
\hline & & & & & Irregular menstruation X07 & $\begin{array}{l}\text { Decoction mixed with lemon Juice } \\
\text { (O) }\end{array}$ \\
\hline Solanaceae & $\begin{array}{l}\text { Datura stramonium L. }+ \\
\text { (PhB106) }\end{array}$ & Tatura (C) & 6.05 & Leaves & Asthma R96 & Fumigation (I) \\
\hline Tamaricaceae & $\begin{array}{l}\text { Tamarix gallica } \mathrm{L} . \\
(\mathrm{PhB} 107)\end{array}$ & Mamey (C) & 4.70 & Aerial parts & $\begin{array}{l}\text { Chills A02, heartburn D03 } \\
\text { Gingivitis D19 }\end{array}$ & $\begin{array}{l}\text { Decoction mixed with honey (O) } \\
\text { Decoction (MW) }\end{array}$ \\
\hline Thymelaeaceae & $\begin{array}{l}\text { Thymelaea hirsuta (L.) Endl } \\
\text { (PhB108) }\end{array}$ & Methnane (A) & 2.18 & Leaves & Skin inflammation S01 & Cataplasm \\
\hline \multirow[t]{3}{*}{ Urticaceae } & $\begin{array}{l}\text { Parietaria officinalis L. } \\
(\mathrm{PhB} 109)\end{array}$ & Talicas (C) & 7.56 & Aerial parts & $\begin{array}{l}\text { Constipation D12, diuretic } \\
\text { U08, urinary calculus U95 }\end{array}$ & \\
\hline & & & & & Haemorrhoids K96 & Cataplasm of juice \\
\hline & Urtica dioica L.+ (PhB110) & $\begin{array}{l}\text { Thimssekssine } \\
\text { (C) }\end{array}$ & 2.85 & Roots & Hair care S24 & $\begin{array}{l}\text { Maceration in water with rosemary } \\
\text { (HW) }\end{array}$ \\
\hline \multirow[t]{3}{*}{ Xanthorrhoeaceae } & $\begin{array}{l}\text { Asphodelus tenuifolius Cav. } \\
\text { (PhB111) }\end{array}$ & Aberouag (C) & 4.87 & Fresh leaves & $\begin{array}{l}\text { Abdominal cramps D01, dia- } \\
\text { betes T10 }\end{array}$ & Decoction $(\mathrm{O})$ \\
\hline & & & & Tubers & Otitis $\mathrm{H} 70$ & Cataplasm \\
\hline & $\begin{array}{l}\text { Tetraena cornuta (Coss.) Beier \& } \\
\text { Thulin (PhB112) }\end{array}$ & Bougriba (A) & $4.2 \mathrm{O}$ & $\begin{array}{l}\text { Flowers } \\
\text { Leaves }\end{array}$ & $\begin{array}{l}\text { Diabetes T10 } \\
\text { Wound healing S18 }\end{array}$ & $\begin{array}{l}\text { Infused honey (O) } \\
\text { Cataplasm }\end{array}$ \\
\hline
\end{tabular}

1. Language: A: Arabic, C: Chaoui. 2. Administration: EC: Eye compress, ED: Eye drop, FB: Footbath, HW: Hair wash, I: Inhalation, MW: Mouth wash, ND: Nose drops, O: Oral, R: Rectal, SB: Sitz bath, T: Topical, VE: Vaginal enema. The species marked in bold are species with a new record of traditional use. *: Species that are used in traditional medicine in Algeria for the first time. $\uparrow$ New use. $\$$ Toxic plant 


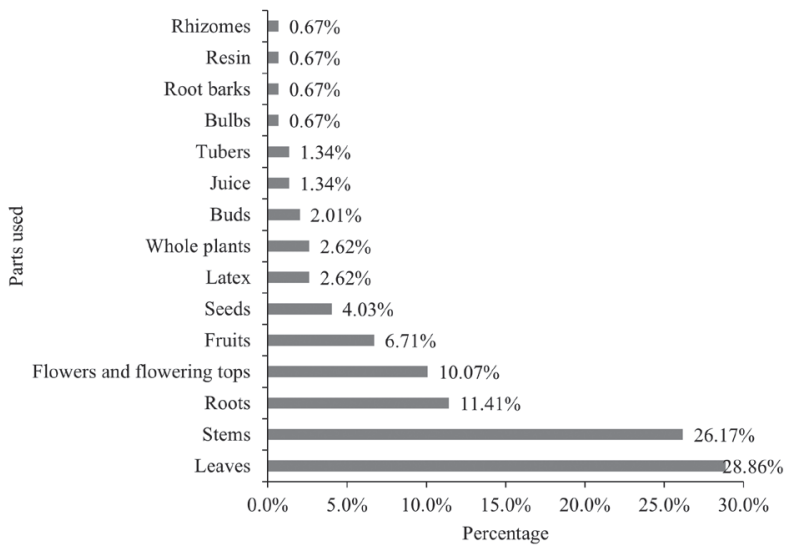

Figure 3: Frequency of particular plant part used in the preparation of medicines.

\subsection{Plant parts used, method of collection and administration}

Concerning the exploited parts of the plants, it emerges from this investigation that all the parts are used (Fig. 3). The most commonly used organs were leaves $(28.86 \%)$ followed in this order by stems (including twigs and stem bark, $26.17 \%$ ), roots $(11.41 \%)$, flowers, and floral tops $(10.07 \%)$, fruits $(6.71 \%)$, and seeds $(4.03 \%)$. All of the remaining organs (latex, whole plant, buds, juice, tubers, bulbs, resin, and rhizomes) did not exceed 12\%. Similar results have been obtained both in Algeria Bouzabata (2013), Hadjaidji-Benseghier \& Derridj (2013), and Chohra and Ferchichi (2019).

Generally, the choice of the plant organ to be used in the therapeutic field comes from its concentration of active ingredients responsible for the medicinal properties of plants (Nacoulma 1996). This author explained the wide use of leaves by the fact that they are the site of photochemical reactions and the reservoir of secondary metabolites. The study by Bitsindou (1986) offers another explanation to this by the relative ease of finding and collecting leaves throughout the year as compared to others part as well as simplicity in remedy preparation.

Turning to the different ways of remedy preparation, various methods were identified, namely decoction, infusion, maceration, juice, cataplasm, fumigation, inhalation, tinctures, suppository, nasal and ophthalmic drops (Fig. 4). The results indicated that the remedies were prepared in the majority from decoction $(37.68 \%)$, infusion $(26.08 \%)$, cataplasm $(19.32 \%)$, chewing $(6.76 \%)$ and maceration $(3.38 \%)$. The percentage of the other methods of preparation grouped (juice, fumigation, inhalation, tinctures, sup- pository, nasal and ophthalmic drops) did not exceed $3 \%$. The use of decoctions and infusions as medicinal preparations has a long tradition among plant users. The reason is that the users consider decoctions and infusions to promote disinfection of the body and keep it warm (Lahsissene \& Kahouadji 2010). In another study, Salhi et al. (2010) believe that infusions and decoctions are excellent extractors of the secondary metabolites responsible for the therapeutic effect of the plant and attenuate or cancel out the toxic effect of certain recipes.

The great majority of the remedies were administrated orally $(57 \%)$ or topically $(38.03 \%)$. The other routes (nasal, ocular, rectal, auricular, and respiratory) are present too but marginally (less than 5\%).

Various ethnobotanical surveys conducted in Algeria (Kadri et al. 2018; Lazli et al. 2019) or elsewhere (Abouri et al. 2012; Jdaidi \& Hasnaoui 2016) have reported similar observations. This result could be explained by the fact that the local population is mainly affected by internal diseases (MSPRH 2008) which could favour oral administration. By elsewhere, Ruiz and Scioli Montoto (2018) believe this route to be the most practical, the safest, and above all, the least expensive. Another possible explanation for this may be related to the addition of some solvents or additives such as water which are believed to increase extraction of bioactive molecules during remedy preparation while minimizing their harmful and toxic effects (Petkova et al. 2020; Redouan et al. 2020).

The majority of the recipes were drawn from a single plant $(81.15 \%, 176$ recipes), some remedies contain two or three plants $(5.09 \%, 11$ recipes) and in some cases, the plants were mixed with honey (7.87\%, 17 recipes), oil (3.24\%, seven recipes), milk

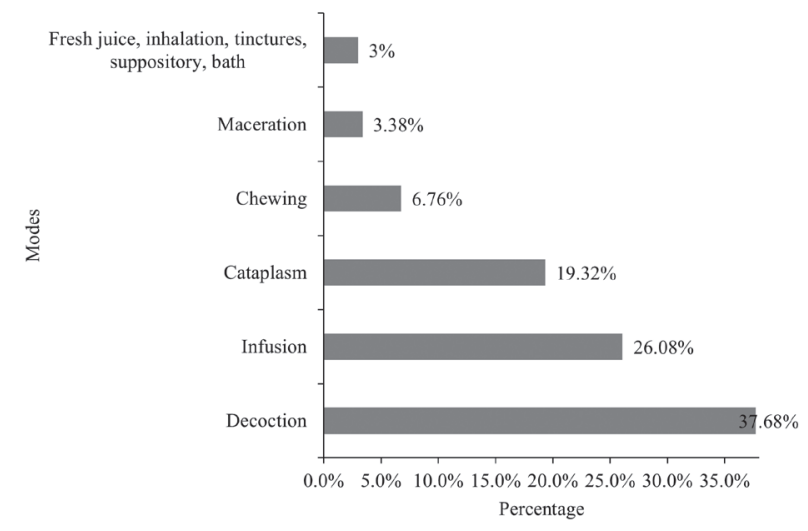

Figure 4: Route of administration of medical plants studied. 
$(0.92 \%$, two recipes) and finally vinegar and butter $(0.46 \%$ and single recipe each). This indicates that monospecific recipes are much easier and quicker to prepare for remedy than the other recipes.

The predominance of single plant recipes has already been reported by Kouadio et al. (2016). These authors estimate that this is beneficial to patients, because of the dangerous nature of plant associations. Also, El-Said et al. (1969) reported 30\% rate of fatal accidents due to the use of mixtures in Africa. However, these findings do not support the previous research of Bussman and Sharon (2006), for whom the use of more than one plant species per recipe is associated with the additive or synergistic effects that they could have while the use of honey or milk seems intended to reduce the bitterness of certain preparations (Tchacondo et al. 2011). Objectively, due to the interactions that can occur between compounds from several plants, recipes made up of more than three plants should be used with caution and sparingly.

Several diseases and dysfunctions treated with medicinal plants were identified during this study (Fig. 5). Plants that treat two ailment categories were the first coming in $(38.39 \% ; 43$ species), followed by plants that treat three ailment categories $(29.46 \%$; 33 species) as well as those who treat one ailment $(20.53 \% ; 23)$ and plants that treat four ailment categories $(10.17 \% ; 12)$. These plants were mainly involved in the treatment of diseases of the digestive system such as indigestion, constipation, diarrhoea, flatulence, colic, and stomachache (23.99\%), skin diseases such as dermatitises, warts and corns $(15.60 \%)$, hormonal disorders, and dysfunctions such as diabetes and menstrual cycle disorders (14.45\%), renal disorders $(10.98 \%)$, in affections of the bronchopulmonary apparatus such as cooling, cough, and cold $(7.51 \%)$ and rheumatism (6.93\%). Other ailments such as venomous bites and stings $(2.89 \%)$, ENT (ear, nose, and throat) diseases (2.31\%), oral diseases (2.02\%), haemorrhoidal disorders $(2.02 \%)$, etc., all recorded an incidence rate which not exceed 3\% for each. These results are identical to those observed by Chohra and Ferchichi (2019) during the work carried out in the neighbouring region of Belezma. Indeed, the most treated pathologies are the same as those observed during our study and this similarity could be explained by the geographical proximity of the two regions and the continuous exchange of therapeutic knowledge between the two populations. However, these values do not fully reflect the health profile of Algeria marked by a dominance of high blood pres-

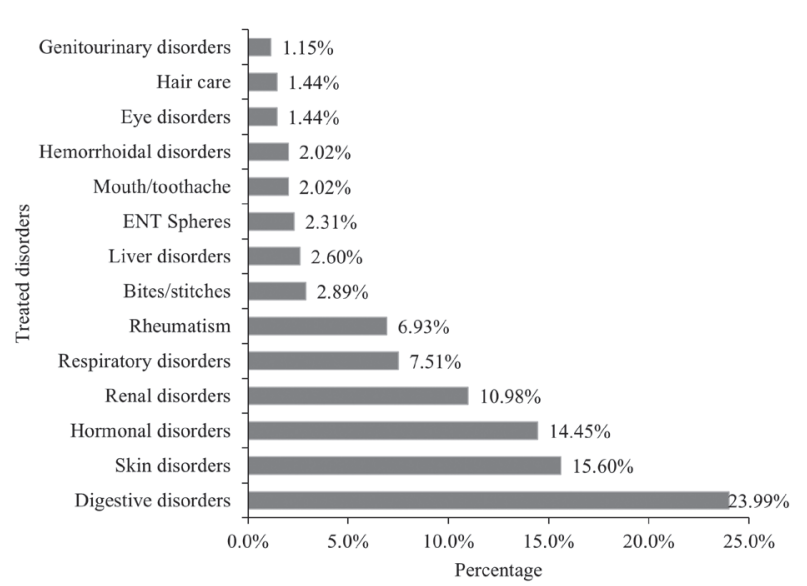

Figure 5: Most frequently treated disorders using medical plants, according to the ICPC 2 classification.

sure, diabetes, and joint diseases (MSPRH 2008). These somewhat different results can be explained by different factors such as the diet in the study area, consisting essentially of excessive consumption of pasta and tobacco which would cause digestive and respiratory diseases (Souilah et al. 2018). According to these same authors, the very cold climate in winter can also explain respiratory diseases while respiratory disorder and skin diseases can be explained through allergies that can occur in contact with plants and pollen emissions.

\section{Conclusions}

This study brings the first ethnobotanical survey in the Aures region in eastern Algeria. It has enabled us to have an overview of the socio-demographic characteristics of the herbal medicine users, to identify the spontaneous medicinal plants growing in the region as well as how treatment is offered within the local populations. This survey revealed that the respondent users seek medicinal plants for preferential treatment. They are between 18 and 82 years of age, mostly female, married, and with little or no education. Also, most of the knowledge is acquired by hereditary transmission.

From an ethnobotanical point of view, local populations use 112 spontaneous medicinal species belonging to 107 genera and 47 families. Among these species, the most important are those that dominate the local flora: Asteraceae, Lamiaceae, Amaranthaceae, Fabaceae, and Poaceae. The most commonly used plants were Rosmarinus officinalis, Pistacia lentiscus, Marrubium vulgare, Pinus halepensis, Artemisia Campestris, Cupressus sempervirens, Artemisia herba-alba, Calendula officinalis, and Juni- 
perus phoenicea and new medicinal uses of 28 plants were recorded for the first time. The aerial organs are the most used parts while the decoction is the most administered method of preparation. Most of the medicinal species identified are used mainly in single plant recipes for the treatment, mainly, of digestive pathologies and skin diseases.

It emerges from this study that the use of traditional medicine based on spontaneous medicinal plants is still very present in the Aures region. This work makes it possible to safeguard the practices of phytotherapy which are in danger of extinction due to the oral transmission of these traditions. It can constitute a starting point for other work in the pharmacological and biochemical fields to enhance the floristic richness of the region.

\section{ACKNOWLEDGMENTS}

The authors are grateful to Melha Baziz for the critical reading of the manuscript and Meriem Hammadi for the translation of the manuscript. We also thank the informants who unreservedly shared with us their knowledge of medicinal plants.

\section{COMPETING INTEREST STATEMENT}

The authors declare no conflict of interest.

\section{REFERENCES}

Abouri, M., El Mousadik, A., Msanda, F., Boubaker, H., Saadi, B. \& Cherifi, K. (2012) An ethnobotanical survey of medicinal plants used in the Tata Province, Morocco. International Journal of Medicinal Plants Research, 1, 99-123. DOI: https://doi.org/10.4314/jab.v84i1.5

African Plant Database (version 3.4.0) (2020) Conservatoire et jardin botaniques de la Ville de Genève and South African National Biodiversity Institute, Pretoria. Retrieved from: http:/www. ville-gen.ch/musinfo/bd/cjb/africa (Accessed 13.11.2020)

Agrawal, D. (1997) Traditional knowledge systems and western science. Current Science, 73, 731733.

Al-Adhroey, A.H., Nor, Z.M., Al-Mekhlafi, H.M. \& Mahmud, R. (2010) Ethnobotanical study on some Malaysian anti-malarial plants: a community-based survey. Journal of Ethnopharmacology, 132, 362-364. DOI: https://doi.org/10.1016/j. jep.2010.08.006

El Alami, A., Loubna, F., \& Chait, A. (2016) Etude ethnobotanique sur les plantes médicinales spontanées poussant dans le versant nord de l'Atlas d'Azilal (Maroc), Algerian Journal of Natural
Products, 4, 271-282. (in French) DOI: https:// doi.org/10.1025/ajnp.2016.4.2.271

Anyinam, C. (1995) Ecology and ethnomedicine: exploring links between current environmental crisis and indigenous medical practices. Social Science \& Medicine, 40, 321-329. DOI: https:// doi.org/10.1016/0277-9536(94)E0098-D

Atmani, D., Chaher, N., Berboucha, M., Ayouni, K., Lounis, H., Boudaoud, H., et al. (2009) Antioxidant capacity and phenol content of selected Algerian medicinal plants. Food Chemistry, 112, 303-309. DOI: https://doi.org/10.1016/j.foodchem.2008.05.077

Baba Aissa, F. (2000) Les plantes médicinales en Algérie. Alger: ed. Bouchène. (in French)

Bahassan, A., Zidane, L, El Yacoubi., H. \& Rochdi., A. (2014). Ethnobotanique et taxonomie des plantes médicinales utilisées pour le traitement des pathologies du système digestif à Hadramaout (Yémen). Phytothérapie, 12, 399-416. (in French) DOI: https://doi.org/10.1007/s10298014-0897-8

Beghami, Y. \& De Bélair, G. (2007). Les groupements végétaux de la cédraie du massif de Chélia «Aurès » Algérie. Bulletin de l'association des botanistes lorrains, numéro spécial 1 : Actes du colloque GEOFLORE 2007, 153159. (in French)

Beghami, Y. (2013) Écologie et dynamique de la végétation de l'Aurès : analyse spatio-temporelle et étude de la flore forestière et montagnarde. $\mathrm{PhD}$ thesis, Université de Biskra, Algérie. (in French)

Beghami, Y., Kalla, M., Vela, E., Thinon, M. \& Benmessaoud, H. (2013). Le Genévrier thurifère (Juniperus thurifera L.) dans les Aurès, Algérie : considérations générales, cartographie, écologie et groupements végétaux. Ecologia mediterranea: Revue internationale d'écologie méditerranéenne $=$ International Journal of Mediterranean Ecology, 39, 17-30. (in French) DOI : https://doi.org/10.3406/ecmed.2013.1288

Beloued, A. (2005) Les plantes médicinales d'Algérie. Alger : ed. Office des publications universitaires (OPU). (in French)

Benmessaoud, H., Kalla, M. \& Driddi, H. (2009) Évolution de l'occupation des sols et desertification dans le sud des Aurès (Algérie). M@ppemonde 94, 1-11. (in French)

Bezza, L., Mannarino, A., Fattarsi, K., Mikail, C., Abou, L., Hadji-Minaglou, F. et al. (2010) Chemical composition of the essential oil of Artemisia herba-alba issued from the district of Biskra (Algeria), Phytothérapie, 8, 277-281. DOI: https:// doi.org/10.1007/s10298-010-0576-3 
Bitsindou, M. (1986) Enquêtes sur la phytothérapie traditionnelle à Kindamba et Odzala (Congo) et analyse de convergences d'usage des plantes médicinales en Afrique centrale. PhD thesis, Université Libre de Bruxelles, Belgique. (in French)

Bouallala, M., Bradai, L. \& Abid, M. (2014) Diversité et utilisation des plantes spontanées du Sahara septentrional algérien dans la pharmacopée saharienne. Cas de la région du Souf. Revue El Wahat pour les Recherches et les Études, 7, 18-26. (in French)

Boutamine, L. (2018) Famille chaouie et interculturalité, Revue Annales du patrimoine, 18, 29-43. (in French)

Bouterfas, K., Mehdadi, Z., Benmansour, D., Khaled, M.B., Bouterfas, M. \& Latreche, A. (2014) Optimization of Extraction Conditions of Some Phenolic Compounds from White Horehound (Marrubium vulgare L.) Leaves. International Journal of Organic Chemistry, 4, 292-308. DOI: https:// doi.org/10.4236/ijoc.2014.45032

Bouzabata, A. (2013) Traditional Treatment of high blood pressure and diabetes in Souk Ahras District. Journal Pharmacognosy and Phytotherapy, 5, 12-20. (in French) DOI : https://doi. org/10.5897/JPP11.065

Bouzabata, A. \& Yavuz, M. (2019) Médecine traditionnelle et ethnopharmacologie en Algérie: de l'histoire à la modernité. Ethnopharmacologia, 62, 86-92. (in French)

Bouzid, A., Chadli, R. \& Bouzid, K. (2017) Étude ethnobotanique de la plante médicinale Arbutus unedo L. dans la région de Sidi Bel Abbés en Algérie occidentale. Phytothérapie, 15, 373378. (in French) DOI: https://doi.org/10.1007/ s10298-016-1027-6

Bussmann, R.W. \& Sharon, D. (2006) Traditional medicinal plant use in Northern Peru: tracking two thousand years of healing culture. Journal of Ethnobiology and Ethnomedicine, 2, \#47 . DOI: https://doi.org/10.1186/1746-4269-2-47

Chaachouay, N., Benkhenigue, O., Fadli, M., El Ibaoui, H. \& Zidane, L. (2019) Ethnobotanical and ethnopharmacological studies of medicinal and aromatic plants used in the treatment of metabolic diseases in the Morroccan Rif. Heliyon, 5, e02191. DOI: https://doi.org/10.1016/j.heliyon.2019.e02191

Chohra, D. \& Ferchichi, L. (2019) Ethnobotanical study of Belezma National Park (BNP) plants in Batna: East of Algeria. Acta Scientifica Naturalis, 6, 40-54.

Cuénod, a., (1954) Flore analytique et synoptique de la Tunisie (Cryptogames Vasculaires, Gymnospermes et Monocotylédones). Tunis: Office de l'expérimentation et de la vulgarisation agricoles de Tunisie. (in French)

Delartigue, R. (1904) Monographie de l'Aurès. Constantine: Marlé-Andrino. (in French)

Dhaou, S.O., Jeddi, K. \& Chaieb, M. (2010) Les Poaceae en Tunisie : systématique et utilité thérapeutique. Phytothérapie, 8, 145-152. (in French) DOI: https://doi.org/10.1007/s10298-010-05331

Djeridane, A., Yousfi, M., Nadjemi, B., Boutassouma, D., Stocker, P. \& Vidal, N., (2006) Antioxidant activity of some Algerian medicinal plants extract containing phenolic compounds. Food Chemistry, 97, 654-660. DOI: https://doi. org/10.1016/j.foodchem.2005.04.028

Dobignard, A. \& Chatelain, C. (2010-2013) Index synonymique de la Flore d'Afrique du nord. Vol 1 : Pteridophyta, Gymnospermae, Monocotyledonae. Genève: Publication hors-série 11, ed des Conservatoire et Jardin botaniques de la Ville de Genève. (in French)

El Rhaffari, L. \& Zaid, A. (2002) Pratique de la phytothérapie dans le sud-est du Maroc (Tafilalet). Un savoir empirique pour une pharmacopée rénovée. In : J. Fleurentin, J. Pelt \& G. Mazars (Eds.) Des sources du savoir aux médicaments du futur (pp. 293-31). Marseille: IRD Éditions. (in French)

El-Said, F., Sofowara, E.A., Malcolm, S.A. \& Hofer, A. (1969) An investigation into the efficacy of Ocimum gratissimum as used in Nigerian native medicine. Planta Medica, 17, 195-200. DOI: https://doi.org/10.1055/s-0028-1099846

Gürağaç Dereli, F. T., Ilhan, M. \& Küpeli Akkol, E. (2020) Identification of the main active antidepressant constituents in a traditional Turkish medicinal plant, Centaurea kurdica Reichard. Journal of Ethnopharmacology, 249, 112373. DOI: https://doi.org/10.1016/j.jep.2019.112373

Hadjaiji-Benseghier, F. \& Derridj, A. (2013) Relative importance of the exploitation of medicinal plants in traditional medicine in the Northeastern Sahara. Emirates Journal of Food and Agriculture, 25, 657-665. DOI: https://doi.org/10.9755/ ejfa.v25i9.14426

Hamel, T., Sadou, S., Seridi, R., Boukhdir, S. \& Boulemtafes, A. (2018) Pratique traditionnelle d'utilisation des plantes médicinales dans la population de la péninsule de l'Edough (nord-est algérien). Ethnopharmacologia, 59, 65-71. (in French)

Hernández, V., Mora, F. \& Meléndez, P. (2012) A study of medicinal plant species and their ethnomedicinal values in Caparo Barinas, Venezuela. Emirates Journal of Food and Agriculture, 24, 128-132. 
ICPC (2015) International Classification of Primary Care (ICPC). Retrieved from http://www.who. int/classifications/icd/adaptations/icpc2/en// (Accessed 15.11.2020)

Jdaidi, H. \& Hasnaoui, B. (2016) Étude floristique et ethnobotanique des plantes médicinales au nord-ouest de la Tunisie : cas de la communauté d'Ouled Sedra. Journal of Advanced Research in Science and Technology, 3, 281-291. (in French)

JORADP (2012) Retrieved from https://www.joradp. dz/FTP/JO-FRANCAIS/2012/F2012003.pdf (Accessed 21.07.2020)

Kadri, Y., Moussaoui, A. \& Benmebarek, A. (2018) Étude ethnobotanique de quelques plantes médicinales dans une région hyper aride du Sud-ouest Algérien « Cas du Touat dans la wilaya d'Adrar ». Journal of Animal \& Plant Sciences, 36, 5844 5857. (in French)

Kouadio, K.H., Gala Bi, T.J., Assiri Assiri, A. \& Yao-Kouamé, A. (2016) Characterization of traditional agroforestry systems of cacao and their impact on some physical fertility parameters of soil in southwestern of Côte d'Ivoire. International Journal of Multidisciplinary Research and Development, 3, 145-152.

Lahsissene, H. \& Kahouadji, A. (2010) Analyse ethnobotanique des plantes médicinales et aromatiques de la flore marocaine : cas de la région de Zaër. Phytothérapie, 8, 202-209. (in French) DOI: https://doi.org/10.1007/s10298-010-0569$\underline{2}$

Lazli, A., Beldi, M., Ghouri, L. \& Nouri, N. (2019) Étude ethnobotanique et inventaire des plantes médicinales dans la région de Bougous (Parc National d'El Kala, Nord-est algérien). Bulletin de la Société Royale des Sciences de Liège, 88, 22-43. (in French) DOI: https://doi. org/10.25518/0037-9565.8429

Mehdioui, R. \& Kahouadji, A. (2007) Étude ethnobotanique auprès de la population de la forêt d'Amisttène : cas de la commune d'Imi n'Tlit (province d'Essaouiria). Bulletin de l'institut Scientifique, Rabat, section sciences de vie, 29, $11-20$.

Mohagheghzadeh, A., Faridi, P., Shams-Ardakani, M. \& Ghasemi, Y. (2006) Medicinal smokes. Journal of Ethnopharmacology, 108, 161-184. DOI: https://doi.org/10.1016/j.jep.2006.09.005

Mokkadem, A. (1999) Cause de dégradation des plantes médicinales et aromatiques d'Algérie. Revue Vie et Nature, 7, 24-26. (in French)

MSPRH. (2008) Ministère de la Sante, de la Population et de la Réforme Hospitalière. Suivi de la situation des enfants et des femmes : enquête na- tionale a indicateurs multiples (MICS3). Alger : MSPRH ed.

Muthu, C., Ayyanar, M., Raja, N. \& Ignacimuthu, S. (2006) Medicinal plants used by traditional healers in Kancheepuram district of Tamil Nadu, India. Journal of Ethnobiology and Ethnomedicine, 2, \#43. DOI: https://doi.org/10.1186/17464269-2-43

Nacoulma, O.G. (1996) Plantes médicinales et pratiques médicales traditionnelles au Burkina Faso: cas du plateau central. $\mathrm{PhD}$ thesis, Université de Ouagadougou, Burkina Faso. (in French)

Olivier de Sardan, J.P. (1995) Anthropologie et développement : essai en socio-anthropologie du changement social. Paris: ed Karthala. (in French)

Ould El Hadj Didi, M., Hadj-Mohammed, M. \& Zabeirou, H. (2003) Place des plantes spontanées dans la médicine traditionnelle de la région de Ouargla (Sahara septentrional Est). Courrier du savoir, 3, 47-51. (in French)

Özdemir, E. \& Alpinar, K., (2015) An ethnobotanical survey of medicinal plants in western part of central Taurus Mountains: Aladaglar (Nigde - Turkey), Journal of Ethnopharmacology, 166, 53-65. DOI: https://doi.org/10.1016/i.jep.2015.02.052.

Petkova, N., Ognyanov, M., Kirchev, M. \& Stancheva, M. (2020) Bioactive compounds in water extracts prepared from rosehip-containing herbal blends. Journal of Food Processing and Preservation, (in press). DOI: https://doi.org/10.1111/ jfpp. 14645

Quezel, P. \& Santa, S. (1962-1963) Nouvelle flore de l'Algérie et des régions désertiques méridionales, Vol $1 \&$ 2. Paris: ed CNRS. (in French)

Rached, W., Benamar, H., Bennaceur, M. \& Marouf, A. (2010) Screening of the Antioxidant Potential of Some Algerian Indigenous Plants. Journal of Biological Sciences, 10, 316-324. DOI: https:// doi.org/10.3923/jbs.2010.316.324

Rebbas, K., Bounar, R., Gharzouli, R., Ramdani, M., Djellouli, Y. \& Alatou, D. (2012) Plantes d'intérêt médicinale et écologique dans la région d'Ouanougha (M'sila, Algérie). Phytothérapie, 10, 131-142. (in French) DOI: https://doi. org/10.1007/s10298-012-0701-6.

Redouan, F.Z., Benìtez, G., Picone, R.M., Crisafulli, A., Yebouk, C., Bouhbal, M. et al. (2020) Traditional medicinal knowledge of Apiaceae at Talassemtane National Park (northern Morocco). South African Journal of Botany, 131, 118-130. DOI: https://doi.org/10.1016/j.sajb.2020.02.004

Ruiz, M.E \& Scioli Montoto, S. (2018) Routes of Drug Administration. In: A. Talevi \& P.A.M. 
Quiroga (Eds.), ADME Processes in Pharmaceutical Sciences: Dosage, Design, and Pharmacotherapy Success (pp 97-133). Cham: Springer International Publishing. DOI: https://doi. org/10.1007/978-3-319-99593-9

Salerno, G., Guarrera, P.M. \& Caneva, G. (2005) Agricultural, domestic and handicraft folk uses of plants in the Tyrrhenian sector of Basilicata (Italy). Journal of Ethnobiology and Ethnomedicine 1, \#2. DOI: https://doi.org/10.1186/17464269-1-2

Salhi, S., Fadli, M., Zidane, L. \& Douira, A. (2010) Études floristique et ethnobotanique des plantes médicinales de la ville de Kénitra (Maroc), Lazaroa 31, 133-146. (in French) DOI: https://doi. org $/ 10.5209 / \mathrm{rev}$ LAZA.2010.v31.9

Sanogo, R. (2006) Le Rôle des Plantes Médicinales en Médecine Traditionnelle. In : 10 ème École d'Été de l'IEPF et du SIFEE (06-10 Juin 2006, Bamako, Mali). Bamako : Université de Bamako (pp. 1-53). (in French)

Souilah, N., Zekri, J., Grira, A., Akkal, S. \& Medjroubi, K. (2018) Ethnobotanical study of medicinal and aromatic plants used by the population National Park of El Kala (north-eastern Algeria). International Journal of Biosciences, 12, 55-77. DOI: https://doi.org/10.12692/ijb/12.4.55- 77

Staub, P.O., Geck, M.S., Weckerle, C.S., Casu, L. \& Leonti, M. (2015) Classifying diseases and remedies in ethnomedicine and ethnopharmacology. Journal of Ethnopharmacology, 174, 514-519. DOI: https://doi.org/10.1016/j.jep.2015.08.051

Su, T., Cheng, B.C., Fu, X.Q., Li, T., Guo, H., Cao, H.H. et al. (2016) Comparison of the toxicities, activities and chemical profiles of raw and processed Xanthii Fructus. BMC Complementary and Alternative Medicine, 16, \#24. DOI: https:// doi.org/10.1186/s12906-016-0994-3

Tabuti, J.R., Dhillion, S.S. \& Lye, K.A. (2003) Ethnoveterinary medicines for cattle (Bos indicus) in Bulamogi County, Uganda: plant species and mode of use Journal of Ethnopharmacology, 88, 279-286. DOI: https://doi.org/10.1016/s03788741(03)00265-4

Tchacondo, T., Karou, D.S., Batawila, K., Agban, A., Ouro-Bang'na, K., Anani, K.T. et al. (2010) Herbal remedies and their adverse effects in Tem tribe traditional medicine in Togo. African Journal of Traditional, Complementary, and Alternative Medicines, 8, 45-60. DOI: https://doi. org/10.4314/ajtcam.v8i1.60522

Trotter, R.T. \& Logan, M.H. (1986) Informant consensus: a new approach for identifying potentially effective medicinal plants. In: N. Etkin (Eds). Plants in Indigenous Medicine and Diet: Behav- ioral approaches (pp. 91-112). 1 st ed. New York: Red grave Publishing Co., Bredford hills. DOI: https://doi.org/10.1016/S0277-9536(98)00181-6

Vela, E. \& Benhouhou, S. (2007) Evaluation of a new hotspot of plant biodiversity in the Mediterranean Basin (North Africa). Comptes Rendus Biologies, 330, 589-605. (in French) DOI: https://doi.org/10.1016/j.crvi.2007.04.006

Whistler, W.A. (1991) Herbal medicine in the Kingdom of Tonga. Journal of Ethnopharmacology, 31, 339-372. DOI: https://doi.org/10.1016/03788741(91)90020-e

WCSP (2020). World Checklist of Selected Plant Families. Facilitated by the Royal Botanic Gardens, Kew. Retrieved from http://wcsp.science. kew.org/. (Accessed 13.11.2020).

World Health Organisation (2013). Stratégie de l'OMS pour la médecine traditionnelle pour 2014-2023 Hong Kong: OMS ed. (in French)

Yahi, N., Djellouli, Y. \& De Foucault, B. (2008) Floristic diversity and biogeography of cedar forests of Algeria. Acta Botanica Gallica, 155, 403-414. DOI: https://doi.org/10.4236/oje.2012.24028 